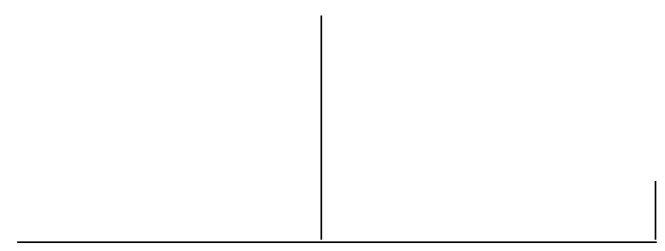

\title{
Psychopathologie de la honte chez le sujet victime de sévices sexuels: Quels enjeux cliniques et thérapeutiques?
}

\author{
Delphine Scotto di Vettimo
}

Le traitement psychothérapeutique des enfants ou des adultes victimes de traumatismes sexuels fait apparaître un sentiment de honte caractéristique. Cet éprouvé de honte, qui empourpre et farde le visage, est d'abord un sentiment social: il apparaît toujours en réaction au regard d'autrui. L'hypothèse ici envisagée est que dans le dispositif psychothérapeutique, l'expression et la reconnaissance de la honte par le sujet, loin d'être des épiphénomènes, constituent un point d'appui essentiel dans l'affirmation et la reconstruction de l'identité. Il s'agit de ne pas réduire la honte à un symptôme mais de l'inclure dans une compréhension plus globale du fonctionnement psychique: se reconnaître et se faire reconnaître sujet honteux, c'est déjà s'affirmer sujet.

L'évocation d'une situation clinique sera proposée pour décrire comment la prise en compte de l'éprouvé de la honte dans une écoute posttraumatique a des effets positifs sur la dynamique psychique du sujet.

Mots clés: Sentiment de honte, trauma sexuel, narcissisme, identité, psychothérapie 


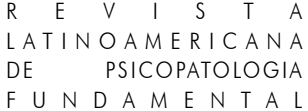

La honte désigne par excellence un type d'affect, non assimilable à l'angoisse, à la fois prévenant, signalant et sanctionnant un franchissement des limites de l'intime, franchissement qui échappe au contrôle de la volonté, corrélatif d'un état d'impréparation et qui dévoile cette part d'intime à l'autre et éventuellement m'en dépossède, au profit de cet autre.

Claude Barazer

\section{Introduction}

Elle. Enfermée dans la survie d'un événement inoubliable, dans la déréliction extrême de ce qu'elle ne parvient pas à mettre en mots, en affects, en images et qui prend dans les entretiens, aujourd'hui encore, la forme d'un silence lourd qui la submerge. Silence qui serait la seule traduction possible de cette souffrance qui la fixe à demeure. Le viol constitue une expérience extrême de violence dans laquelle l'atteinte à l'identité sexuée, à l'intimité et à la dignité humaine relève d'un informulable, et vient littéralement abraser l'inscription du sujet dans son ontologique et humaine condition. Une souffrance qui va trouver dans l'épreuve de honte son registre le plus singulier: il s'agit là d'une formulation capitale, très précisément quant à la question traumatique et à son élaboration psychique. C'est à partir de l'expression et de la reconnaissance d'un sentiment de honte qu'elle pourra commencer à faire partager au clinicien l'événement traumatique survenu cinq ans plus tôt. Autrement dit, l'accession au traumatisme peut se manifester d'abord par la capacité à énoncer la honte qu'il a engendré chez le sujet.

La honte! Difficile d'aborder, et à fortiori d'approfondir, un sujet qui, par définition, suscite un malaise. Qu'est-ce que la honte? Quel est son mécanisme psychique? Quel type d'incertitude narcissique imaginaire provoque-t-elle?

"Avoir honte", "Avoir la honte", "être honteux" ou encore "faire honte": ces formulations, bien que classiques, n'appartiennent pas au vocabulaire spécifique de la psychopathologie ou de la psychanalyse. À ce sujet, Rycroft (1982), auteur d'un dictionnaire de langue anglaise, écrit que "la honte est la Cendrillon des émotions désagréables 


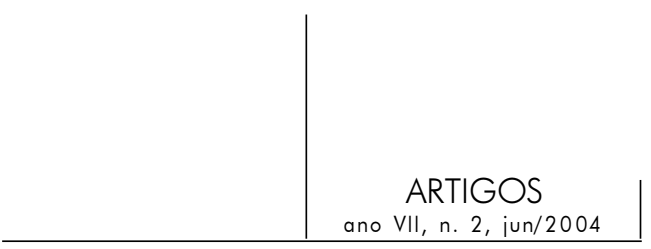

car on y a prêté beaucoup moins d'attention qu'à l'angoisse, la culpabilité, la dépression". S'ouvrent ici les prémices d'une question fondamentale qui porte sur le statut métapsychologique de la honte et plus largement, dans le cadre de la pratique clinique, sur la co-occurrence entre traumatisme sexuel et sentiment de honte. L'argument ici proposé situe l'expérience de honte comme intrinsèque au traumatisme sexuel. En effet, j'ai été tout particulièrement sensibilisée à l'expression de sentiments de honte qui colorent spécifiquement le récit d'expériences traumatiques sexuelles, et qui envahissent l'ensemble de la vie psychique des sujets. En outre, cette problématisation s'argumente de la récurrence, de la persistance de la honte qui devient répétition dans la mise en récit des situations à l'origine de la honte, récits imprégnés de malaises visibles d'où transpirent effroi et désarroi. Le fait traumatique sexuel fige hermétiquement le temps, le cristallise, le gèle et assujettit le sujet à l'achoppement de l'impossible historicisation qui fait symptôme dans les compulsions de répétition. Cette manière d'aborder le problème de la violence sexuelle amène donc à prendre en considération l'acuité de l'expérience de honte inhérente à tout traumatisme sexuel, à la repérer à partir de la pratique clinique portée par un cas, à initier un dispositif transférentiel qui irait de l'expression et de la reconnaissance de la honte par le sujet, à sa subjectivation dans le transfert. En effet, le sujet est d'abord dans une quête désespérée de reconquête et de reconnaissance de la dimension ontologique et survivante de son être.

Le langage est affaire de conventions et d'usages multiples. $\mathrm{Si}$, pour le sujet, l'expression de la honte est porteuse d'une acception précise, elle est pour le clinicien d'abord indice et messagère d'une signification particulière donnée à la parole. Cette interrogation va trouver certains prolongements du point de vue de son usage dans le discours, et plus précisément dans la distinction à opérer entre les propos du sujet où figure le mot honte, et le sentiment de honte. En effet, l'acte de parole peut se distinguer de ce qu'il est censé évoquer et signifier, et l'usage du terme de honte par le sujet ne préjuge pas de l'existence de l'affect: "Si elle est exposition, exhibition, la parole se différencie radicalement de ce qu'elle évoque: on ne peut pas s'asseoir sur le mot chaise" (Jacobi, 2000), autrement dit le mot n'est pas la chose. Dans la mesure où l'acte de parole révèle de lui-même une discontinuité fondamentale avec ce qu'il évoque, il sauvegarde en quelque sorte le corps, le maintient à distance, distance qui est totalement bafouée et abolie dans les actes de violence sexuelle. Dès lors, la question qui, pour le clinicien, se pose, est bien justement, dans le travail clinique, de discerner l'épreuve de honte d'un sujet au delà des termes du discours qui accompagne cette épreuve. Il est vrai que l'usage de ce terme ne renvoie pas à un diagnostic, à une nosographie. Mais il mérite à s'expliquer sur le saut qui consiste à affirmer la présence de honte pour un sujet à partir de l'occurrence de ce terme dans son discours. Enfin, il y 


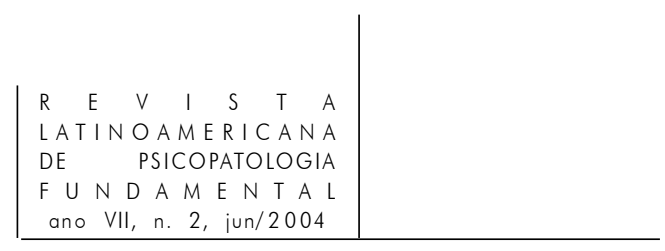

a lieu de situer la honte dans le transfert, dans l'adresse à un interlocuteur, ce que nous aurons l'occasion de développer à partir de l'extrait clinique précité.

Dans un travail de recherche (Scotto di Vettimo, 2001) consacré à la honte, j'ai avancé l'idée suivante: face à l'immaîtrisable du traumatisme, l'expérience de honte témoigne d'une tentative de subjectivation là où s'exhibe, béant, le trou du trauma. Elle vient comme marque d'appartenance humaine là où la rencontre traumatique est venue confronter le sujet au réel de la mort, de l'indicible, de l'insoutenable. En ce sens, et ce fut l'argument princeps de ce travail, la honte déjoue la pulsion de mort à l'œuvre dans la compulsion de répétition. Elle est un essai pour dépasser le trauma, un essai différent de la répétition et plus salutaire aussi, puisqu'elle permet au sujet de continuer à s'éprouver comme tel.

Étymologiquement, "honte" vient du francique haunita, même radical que honnir (1080) qui signifie "mépriser", et revêt une pluralité d'acceptions:

1. Indignité qui inflige un déshonneur humiliant,

2. (1273). Sentiment pénible de sa bassesse, de son déshonneur, de sa confusion, de son abaissement devant les autres, ou simplement de son ridicule,

3. (1611). Sentiment de gêne, de malaise, provoqué par la timidité, la modestie, le manque d'assurance, la crainte,

52 4. Avoir du remords, être dégoûté de, être gêné de.

La honte, qui empourpre et farde le visage, est d'abord un sentiment social: elle apparaît le plus souvent en réaction au regard d'autrui et vient marquer l'échec de la confirmation narcissique. Cette primauté du regard dans l'expérience de honte, trouverait son hypothèse interprétative dans le fait que la honte prendrait sa source dans le regard de l'Autre, regard qui révélerait au sujet ses propres limites, son incomplétude, autrement dit dévoilerait le décalage insoutenable entre l'image narcissique de soi faite de perfection et une image reconnue dans le regard de l'Autre, d'insuffisance et d'imperfection. Nous nous situons là dans la perspective lacanienne classique qui postule, au sujet de l'Autre, que “... c'est de lui que le sujet se constitue, par quoi c'est de l'Autre que le sujet reçoit même le message qu'il émet" (Lacan, 1960, p. 807). L'approche phénoménologique de l'épreuve de honte montre qu'il se produit un instant de choc, un temps d'arrêt sur une image honteuse de soi qui provoque un état de confusion plus ou moins violent chez le sujet, venant l'ébranler dans ses assises narcissiques. "La décharge fulgurante" de honte telle qu'elle a été décrite chez Sartre dans cette perspective, témoigne fort justement de cette expérience émotionnelle où une part du plus intime de soi se trouve brutalement exposé, véritable mise à nu du sujet qui le confine dans un chaos narcissique. Dans l'expérience de honte, l'effet de transparence ressenti en est sans doute la traduction la plus explicite, qui ne saurait mieux s'exprimer que dans l'expression langagière, assez coutumière, de mise à nu. De 


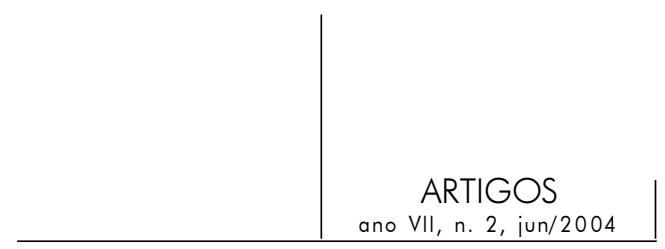

ce point de vue, la honte réduit le sujet à une position d'objet alors qu'un pouvoir excessif est attribué au regard de l'autre, pouvoir d'accéder, dans une dimension scopique extrêmement forte, à son intimité. En d'autres termes, dans l'expérience de honte se produit une défaillance du moi, qui signale et marque le dépassement d'un seuil "... où une part du plus intime de moi se trouve brusquement offerte 'en pâture' à la jouissance d'autrui" (Barazer, 2000, p. 9). Ce ravalement à une fonction d'objet engendre un grand désarroi chez le sujet, car il est alors en proie à une incertitude narcissique angoissante.

L'articulation de la honte au narcissisme va constituer le point de départ et d'appui de ma réflexion. Mon intérêt pour les modalités cliniques et théoriques de la honte dans ses aspects pathologiques et son traitement psychothérapique m'ont orientée vers l'exploration de son économie dans ses liens profonds avec le narcissisme, l'Idéal du Moi et le Moi-Idéal. En tant qu'éprouvé narcissique, la honte est du côté des représentations de l'échec, de l'infériorité, des insuffisances du Moi dans sa confrontation à l'Idéal du Moi et au Moi-Idéal. La honte ne se rattache pas à une faute mais à l'échec de la confirmation narcissique. Dans une perspective topique, c'est dans l'écart implicite entre le Moi, l'Idéal du Moi et le Moi-Idéal que s'éprouve la honte, plus précisément lorsque le Moi n'est pas à la hauteur des exigences issues de l'Idéal du Moi et du Moi-Idéal.

Alors que la honte concerne les instances psychiques de l'Idéal du Moi et du Moi-Idéal, la culpabilité, elle, concerne le Surmoi. Je citerais ici Miollan (1998, p. 164), lorsqu'il écrit: "Le défaut ou le manque engendre la honte; le manquement, la faute, engendrent la culpabilité. On peut éprouver de la honte sans culpabilité lorsque, par exemple, la honte est l'effet d'une souillure limitée. A contrario, la culpabilité peut être ressentie sans honte, comme dans le deuil'"

Autrement dit, la culpabilité est rattachée à l'objet, au surmoi et à l'intériorisation des interdits, implique le plus souvent auto-accusations et autoreproches et se rattache à l'idée de faute, mais, secrète ou pas, en rapport ou non avec des contenus fantasmatiques, le sujet n'est aux prises qu'avec lui-même. Enfin, si la culpabilité est facilement confiée et si sa mise en récit revêt une fonction libératrice (cathartique), la honte est soumise au processus inverse: elle impose un huis clos et condamne au silence puisqu'elle est vécue comme étant irrévocable, le sujet considérant qu'il ne peut que la subir et se cacher. Ainsi, la honte constitue une expérience subjective singulière, celle d'un sujet brusquement dévoilé dans son intimité de pensée, par la présence d'un regard dont il est impossible de se dérober. En ce sens, le sentiment de honte est paradoxal dans son essence même, puisque d'emblée social, étroitement lié au regard de l'autre, tout autant qu'expression de la manifestation d'une revendication singulière.

Mobilisant notre expérience intime, essentiellement sur le versant de l'identification imaginaire (avoir honte pour l'autre), la honte se redouble aisément 


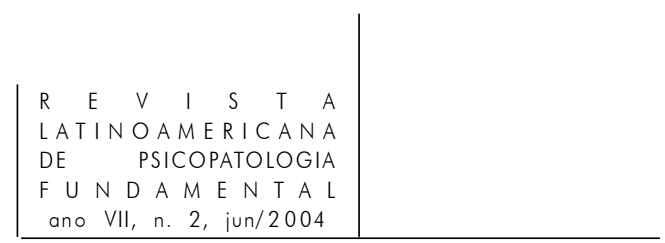

en "honte de la honte" et suspend la parole singulière du sujet. Car c'est la honte elle-même qui interdit sa propre expression. Mais peut-être ce spectre de la honte est-il toujours là, déjà là, avant même le surgissement potentiel de toute indécence, comme une mémoire de cette période de l'enfance, où le jeune enfant attribue sans conteste - au regard des adultes la faculté de lire dans ses pensées. Période également où l'adulte vient prohiber "l'ivresse exhibitionniste" comme c'est le cas pour ce jeune enfant dont parle Freud (1900) à propos de la honte, dans le chapitre consacré aux rêves typiques (le rêve de confusion à cause de la nudité); il découvre à l'origine de ces productions oniriques un souvenir de la prime enfance où "en ce temps-là nous n'avions pas honte d'être nus" (p. 213). L'enfant, dépourvu de pudeur, pervers polymorphe, est mû par ses instincts de petit homme à la découverte du monde. Non conscient de ses poussées instinctuelles, il prend plaisir à s'exhiber. Freud n'a pas manqué d'indiquer la parenté entre les rêves typiques d'exhibition et cette période de l'immoralité enfantine qui ignorait la honte.

Oser dire, oser se raconter, en parler ou s'y dérober, c'est dans le contexte psychothérapeutique ou psychanalytique que le sujet pourra se confronter à ce qui fait honte en lui. Toutefois, cette reconnaissance ne met pas un terme au paradoxe de la honte, à savoir qu'il isole le sujet tout en assurant la sauvegarde d'un lien là où il menace d'être rompu. La honte, tout en étant déstructurante, contribue à sauvegarder l'unité du sujet. Elle apparaît comme tentative ultime d'éprouver une identité. Mais la honte engendre aussi et surtout une souffrance qui bouleverse l'individu dans son fonctionnement psychique et social.

Cette réflexion porte sur les empreintes et les conséquences, sur le fonctionnement psychique, de la confrontation massive à l'expérience de honte, à travers des situations de violences sexuelles, d'humiliation, de déchéance et d'atteintes à la dignité, directement vécues et subies par ces sujets. En d'autres termes, le traitement psychothérapeutique des enfants ou des adultes victimes de traumatismes sexuels fait apparaître un sentiment de honte caractéristique. Pourquoi le traumatisme sexuel produit-il de la honte? Quelles insuffisances et quelles limites cette situation révèle, réactive, soulève? La première hypothèse ici envisagée prend en compte les deux instances psychiques de la personnalité que sont l'Idéal du Moi et le Moi-Idéal, dont les éléments déterminants en sont: d'une part le rapport de l'Idéal du Moi avec l'expérience d'une atteinte sexuelle et de la blessure narcissique irréversible qu'elle provoque, d'autre part le Moi-Idéal en rapport avec la déchéance ressentie dans la honte consécutive au traumatisme sexuel, honte de n'être que la chose, l'objet, le déchet de l'agresseur. La deuxième hypothèse de la honte postule que dans le dispositif psychothérapeutique, l'expression et la reconnaissance de la honte par le sujet, loin d'être des épiphénomènes, constituent un point d'appui essentiel dans l'affirmation et la reconstruction de l'identité. Il s'agit de ne pas réduire la honte à un symptôme 


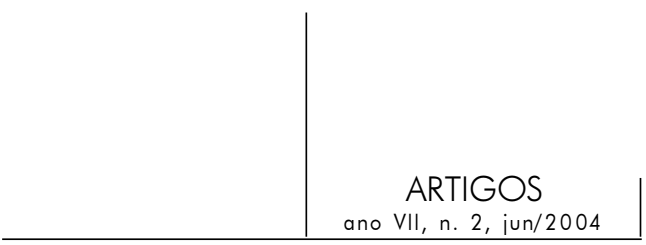

mais de l'inclure dans une compréhension plus globale du fonctionnement psychique: se reconnaître et se faire reconnaître sujet honteux, c'est déjà s'affirmer sujet.

Ici, il faut s'arrêter un instant sur la notion d'affect qui est contemporaine de la naissance même de la psychanalyse, Freud proposant une première classification des névroses selon la manière dont un sujet se comporte au regard des affects. Dans son article sur "L'inconscient" (1915), il définit l'affect de cette façon: “... les affects et sentiments correspondent à des processus de décharge dont les manifestations finales sont perçues comme sensations" (p. 84). Plus précisément, l'affect est défini comme la traduction subjective de la quantité d'énergie pulsionnelle et de ses multiples variations. C'est-à-dire que Freud opère une distinction entre l'aspect subjectif de l'affect et les processus énergétiques qui le conditionnent. De cette conception structurelle résulterait toute une compréhension de la notion de honte comme manifestation subjective et comme relevant de l'identification imaginaire et du narcissisme spéculaire. Dans l'œuvre freudienne, le concept d'identification est défini comme une opération par laquelle le sujet humain se constitue: l'identification aspire à “... rendre le moi propre semblable à l'autre pris comme modèle" (Freud, 1921, p. 169). Cette évolution est corrélative du complexe d'Edipe, dont les effets sur la structuration du sujet sont décrits en termes d'identification, les investissements sur les parents étant abandonnés et remplacés par des processus identificatoires. Cette opération est également marquée du remaniement apporté par la seconde topique de l'appareil psychique, où les instances qui se différencient à partir du ça sont spécifiées par les identifications dont elles dérivent. Ce concept d'identification se trouvera enrichi par différents apports, dont celui de narcissisme, que Freud introduit dans le texte intitulé "Pour introduire le narcissisme" (1914) en envisageant tout particulièrement les investissements libidinaux. Il en fait un processus de structuration fondamental du sujet et le décrit en terme de forme d'investissement pulsionnel. Dans un premier temps, le narcissisme primaire désigne cet état primitif, celui de l'enfant qui se prend lui-même comme objet d'amour. Dans un deuxième temps, Freud décrit la dialectique qui va relier le choix d'objet narcissique, où l'objet est choisi sur le modèle de la personne propre, et l'identification, le sujet se constituant sur le modèle de ses objets antérieurs, notamment parentaux. Le souci majeur de Freud est de mettre en évidence le narcissisme comme une forme d'investissement pulsionnel nécessaire à la vie subjective, comme une donnée structurale du sujet. Il représente “... une sorte d'état subjectif, relativement fragile et facilement menacé dans son équilibre" (Chemama, 1993, p. 200). Ce bref rappel du rôle du narcissisme dans la psychogenèse de l'enfant montre que chaque être investit son moi grâce aux investissements dont il a pu être l'objet dans les premières années de sa vie. 


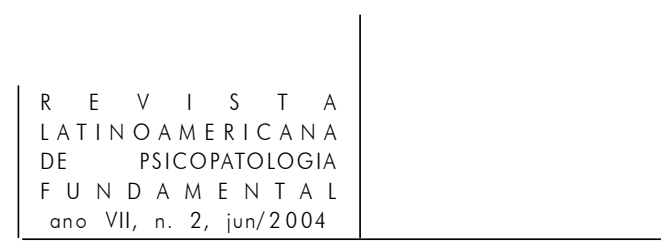

Dans le cadre de la théorie psychanalytique, Lacan a mis en rapport ce moment inaugural de la formation du moi avec cette expérience narcissique fondamentale qu'il désigne sous le nom de stade du miroir, qui marque une étape génétique et ontologique fondamentales, dans la mesure où va s'y constituer la première ébauche du moi. Face au miroir, l'enfant va anticiper, dans un leurre spéculaire et imaginaire, l'appréhension et la maitrise de son unité corporelle. Son comportement face à son image dans le miroir se caractérise par "L'assomption jubilatoire de son image spéculaire par l'être encore plongé dans l'impuissance motrice et la dépendance du nourrissage qu'est le petit homme à ce stade infans ..." (Lacan, 1945a, p. 94). L'enfant perçoit dans l'image du semblable ou dans sa propre image spéculaire une forme (Gestalt) dans laquelle il va anticiper imaginairement l'appréhension et la maîtrise de son unité corporelle (Laplanche e Pontalis, 1967, p. 452). Cette unification va s'effectuer par l'identification de l'enfant à cette image: "Il y suffit de comprendre le stade du miroir comme une identification au sens plein que l'analyse donne à ce terme: à savoir la transformation produite chez le sujet, quand il assume une image" (Lacan, 1945a, p. 94). C'est donc à partir de l'autre semblable et de sa reconnaissance que l'instance du moi s'ébauche et se constitue. Cette expérience narcissique fondamentale constitue la matrice symbolique de ce qui sera le moi. Elle est au fondement de l'expérience imaginaire du moi, constitué d'emblée comme "je-idéal" et "souche des identifications secondaires" (ibid.). Le début de cette structuration subjective caractérise le narcissisme primaire, c'est-à-dire l'investissement pulsionnel du sujet sur lui-même, sur cette image de lui à laquelle il s'identifie. En ce sens, la relation intersubjective, qui est marquée des effets du stade du miroir, est d'abord une relation imaginaire et duelle, où le moi est constitué comme un autre et autrui comme alter ego.

Le registre imaginaire ${ }^{1}$ représente, avec le réel et le symbolique, l'un des trois registres fondamentaux du champ psychanalytique lacanien. Dans une dimension intrasubjective, l'imaginaire concerne le rapport fondamentalement narcissique du sujet à lui-même, rapport où “... l'individu humain se fixe à une image qui l'aliène à lui-même, c'est là l'énergie et c'est là la forme d'où prend origine cette organisation passionnelle qu'il appellera son moi" (Lacan, 1948, p. 113). À considérer cette expérience fondamentale, du point de vue de la relation intersubjective, il n'y a de semblable, c'est-à-dire un autre qui soit moi, que

1. L'imaginaire va résulter de la jonction entre le réel et le symbolique. C'est lors du stade du miroir, précisément au moment de l'assomption jubilatoire de l'enfant au miroir que l'imaginaire connaît son moment inaugural. C'est dans cette dialectique que Lacan (1975-1976) écrit: "Il faut un minimum d'imaginaire pour symboliser le réel". 


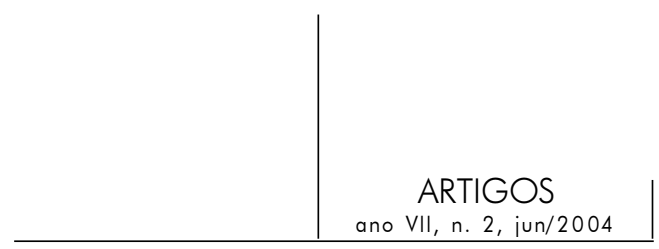

dans la mesure où le moi est originellement un autre. Le registre moïque procède de cette méconnaissance radicale, de cette aliénation, de cette agressivité et de l'amour dans la relation au semblable, relation duelle qui est initialement fondée et captée par l'image d'un semblable (moi-spéculaire) et “... l'identification primaire qui structure le sujet comme rivalisant avec soi-même" (p. 117). Pour Lacan, toute relation imaginaire est vouée à ce leurre: "Je est un autre" (p. 118) écrit-il reprenant le poème de Rimbault. Sur la base de cette identification princeps vont se succéder et s'intercaler les multiples identifications, imaginées et imaginaires, par où le sujet va constituer et enrichir son moi: "Mais fondamentalement ce moi, ou cette image qu'est le moi, est 'extérieur' au sujet et ne peut donc avoir la prétention de le représenter complètement à lui-même" (Chemama, 1993, p. 201). De là va procéder le narcissisme secondaire comme corollaire de cette opération, par où le sujet commence à investir des objets extérieurs à lui, objet différent et distinct de lui et à la fois un objet qui le représente “... puisque c'est son propre moi, un objet qui est l'image pour 'laquelle il se prend', avec tout ce que ce processus comporte de leurre, d'aveuglement et d'aliénation" (ibid.). L'édification de l'idéal $d u$ moi - à partir de ce leurre - représente l'instance de la personnalité qui, dans une dimension symbolique, a la charge de réguler la structure imaginaire du moi et la panoplie des identifications qui s'y déploient, ainsi que l'ensemble des conflits qui animent et régissent, dans une dynamique intersubjective, les rapports du sujet à ses semblables.

Le registre du réel ${ }^{2}$ ne peut être défini que par rapport au symbolique et à l'imaginaire. Il se caractérise d'un défaut fondamental: il est inconnaissable et non symbolisable. La honte participe de ce registre, elle se spécifie de sa rencontre avec le réel. C'est là qu'elle s'éprouve, dans son lien à l'originaire et dans le rapport scopique à l'autre semblable: le réel saisi dans le regard d'autrui. La honte, c'est du pur réel, c'est-à-dire qu'elle surgit de cette réalité qui n'est pas ordonnée par le symbolique. Dans l'expérience de honte, la mise à nu du sujet sous le regard d'autrui invoque ce réel, par où “... il revient dans la réalité à une place où le sujet ne le rencontre pas, sinon sous la forme d'une rencontre qui réveille le sujet de son état ordinaire" (Chemama, 1993, p. 278). Les manifestations physiques, corporelles et physiologiques de la honte témoignent de cette effraction qui fait la chute du sujet dans un réel non symbolisable, signant du même coup l'échec de toute symbolisation.

Le registre symbolique ${ }^{3}$ marque l'affranchissement, chez l'enfant, de la capture imaginaire dans laquelle il se trouvait, depuis l'origine, inscrit. Au temps

2. Le réel incarne et représente la sphère somatique du sujet, la "chair".

3. Le symbolique représente la nomination, par l'Autre, du fonctionnement biologique. 


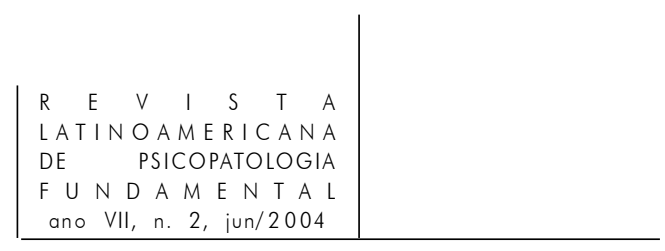

pré-spéculaire où l'enfant se vit comme morcelé, succède le stade où la constitution du moi s'unifie dans la dépendance d'une identification aliénante à l'image spéculaire (siège de la méconnaissance). Mais pour que le petit homme puisse s'approprier et intérioriser cette image, l'Autre (incarné par la mère) doit le reconnaître et le confirmer dans son existence de sujet. Ce signe de reconnaissance va fonctionner comme trait unaire, ${ }^{4}$ c'est-à-dire comme signifiant à partir duquel va pouvoir se constituer l'identification symbolique et l'ébauche de l'idéal du moi: “... il n'y a d'accession à l'identification de l'idéal du moi qu'une fois le terme du grand Autre entré en ligne de compte" (Lacan, 1957-1958, p. 312). Le trait unaire est un repère symbolique qui va soutenir l'identification imaginaire, lorsque l'image spéculaire de l'enfant se regardant dans le miroir sera saisie et appréhendée dans le champ de l'Autre. Le grand Autre, c'est “... le lieu, le siège, le témoin, auquel le sujet se réfère dans son rapport avec un petit $a$ quelconque, comme étant le lieu de la parole" (ibid.). Ainsi, du fait que le sujet se perçoit à travers une image de lui-même qui est projetée et enrichie d'une pluralité de représentants, le "Moi ne peut prendre sa valeur de représentation imaginaire que par l'autre et au regard de l'autre" (Dor, 1995, p. 156). L'image spéculaire à laquelle l'enfant s'identifie est possible dans la mesure où elle passe par la reconnaissance de l'Autre maternel: “... l'échange des regards, manifeste à ce que l'enfant se retourne vers celui qui de quelque façon l'assiste, fût-ce seulement de ce qu'il assiste à son jeu" (Lacan, 1966, p. 70). Ainsi, le jeune enfant ne peut se reconnaître et s'identifier à sa propre image qu'à la condition que l'autre l'identifie et le reconnaisse comme tel. C'est par le moi, instance de l'imaginaire (au sens de l'image) que le sujet se perçoit et s'éprouve: "Le moi, c'est l'image du miroir en sa structure inversée" (Chemama, 1993, p. 278). Il représente l'instance du registre imaginaire, des identifications et du narcissisme. Dans les rapports du sujet à ses semblables se déploie une véritable captation imaginaire du double dans la mesure où le sujet se confond avec son image. En effet, originellement, c'est comme un autre - l'autre du miroir en sa structure inversée - que l'enfant se constitue (narcissisme primaire) et c'est la nomination de l'enfant par la mère, une mère dont le regard le regarde, qui permettra l'accès de l'enfant au registre symbolique (narcissisme secondaire). C'est là tout le champ du narcissisme comme fondateur de l'image du corps de l'enfant et de son statut narcissique, à partir de ce qui est d'abord amour de la mère et ordre du regard porté sur l'enfant.

4. Le trait unaire est "Un concept introduit par Lacan, à partir de Freud, pour désigner le signifiant sous sa forme élémentaire et pour rendre compte de l'identification symbolique du sujet". Chemama, 1993, p. 334. 


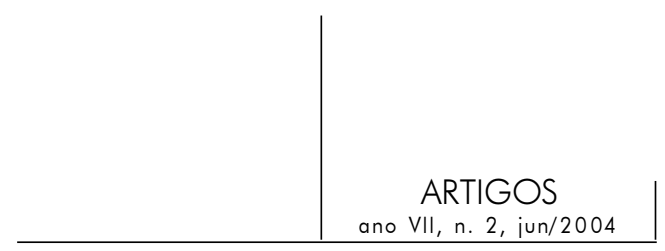

À l'appui de ces considérations et dans une perspective métapsychologique, nous dirons que la honte comme éprouvé narcissique est directement en lien avec l'Idéal du Moi et le Moi-Idéal. Le travail psychothérapeutique auprès de sujets souffrant de honte montrent "l'état singulier de déficit narcissique" (Jacobi, 1998, p. 25). Dans ce sens, la clinique de la honte concerne directement le narcissisme et plus précisément la honte relève d'un fonctionnement sur le mode narcissique. C'est à partir d'une évocation clinique que va être mis à l'épreuve ce corpus d'hypothèses. Mais au préalable, nous proposons de resituer d'abord l'apparition de la honte dans la théorie freudienne. Nous allons voir que la honte entre dans le cadre des affects négatifs avec la culpabilité et l'angoisse, ce qui met en évidence l'existence de liens qui demandent une analyse avec les représentations conscientes, préconscientes et inconscientes. Comment Freud a-t-il situé la honte dans l'ensemble du fonctionnement psychique et en particulier dans la topique telle qu'il l'élaborait au moment même où il tentait de définir cet affect?

\section{Repères psychanalytiques freudiens de la honte}

Dans le texte de la Genèse,$^{5}$ la naissance du sentiment de honte est contemporaine de la découverte de la nudité: Adam et Ève, après avoir commis le péché originel, se découvrirent nus... et honteux. La théorie psychanalytique freudienne fera du texte biblique le point de départ du sentiment de honte, comme celui de l'angoisse et de la culpabilité: "C'est pourquoi - note Freud dans L'interprétation des rêves (1900) - dans le paradis les hommes sont nus et n'ont point de honte, jusqu'au moment où la honte et l'angoisse s'éveillent, où ils sont chassés et où commencent la vie sexuelle et la civilisation" (p. 213).

Selon l'hypothèse phylogénétique freudienne, le mythe de la Genèse inaugure la naissance du sentiment de honte, qui passe par le regard sur "les parties honteuses" et l'introduction de la différence des sexes. La honte émerge en corollaire d'un regard qui met à nu et qui conduisent Adam et Ève à se cacher à la vue de Yahvé Dieu parmi les arbres du jardin d'éden.

5. "L'Ancien Testament". In: La Bible de Jérulasem, p. 31-83.

6. 'En se verticalisant, l'être humain met à découvert ce qui était maintenu caché, 'recouvert' (gedeckt), dans la position courbée. D'où l'association de ces deux termes : les organes génitaux deviennent solidairement sichtbar und schutzbedürftig (visibles et exigeant une protection). Apparaît ainsi le fait d' 'avoir honte' (das Schämen), ce terme pouvant aussi être traduit par 'pudeur'”. Schneider, 1996, p. 22. 


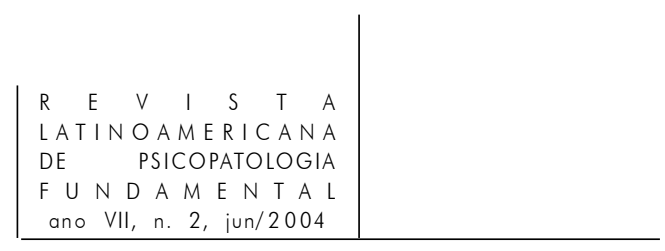

L'arbre de la connaissance du bien et du mal était un privilège que Dieu se réservait et que l'homme a usurpé par le péché originel qui constitue un véritable affront à la souveraineté de Dieu, une faute d'orgueil. Cette révolte s'est exprimée concrètement par la transgression d'un précepte posé par Dieu et représenté sous l'image du fruit défendu. Le serpent symbolise l'adversaire, l'ennemi de Dieu et de l'homme, dans lequel la tradition chrétienne à reconnu le Diable. L'éveil de la concupiscence est la première manifestation du désordre que le péché introduit dans l'harmonie de la création. Adam et Ève, coupables du péché originel, ont donc honte de leur nudité. Ils se retrouvent brusquement confrontés à la différence des sexes, au désir sexuel comme effraction et brèche fondamentale dans le narcissisme et l'état idéal du Paradis terrestre.

En un sens large, le mot allemand Scham désigne dans l'usage freudien, aussi bien la honte comme formation réactionnelle dans la névrose obsessionnelle, comme "digue psychique" avec le dégoût et l'exigence d'idéal esthétique et moral, et dont la mission est de faire rempart à l'envahissement pulsionnel, notamment des pulsions sexuelles de voyeurisme et d'exhibitionnisme au début de la période de latence. Enfin, dans le cadre de la seconde théorie de l'appareil psychique, le dégagement de la notion de sentiment de culpabilité devait conduire Freud à opérer une distinction théorique avec le sentiment de honte, et à spécifier le rapport dialectique entre ces deux notions. L'histoire de la pensée freudienne est complexe et l'étude des textes ne permet pas de localiser une acception du concept de honte précise et déterminée, dans la mesure où le terme allemand est tantôt traduit par "honte" et tantôt par "pudeur", deux équivalents sémantiques qui ne permettent pas de conclure à un usage absolument univoque.

Dès ses premiers écrits, Freud (1905) assimile la honte à l'action des forces refoulantes - répressives - qui ont pour visée de lutter contre le surgissement des pulsions. Dans plusieurs textes, l'auteur cite simultanément "le dégoût, la honte, la moralité" (p. 101). Ce qui était initialement objet de plaisir - plaisir directement lié à la dimension sexuelle sous-jacente - devient, sous l'effet du refoulement et des formations réactionnelles, objet de pudeur, de dégoût ou de honte. Dans son article intitulé "Nouvelles remarques sur les psychonévroses de défense", Freud (1896) écrit:

Alors un reproche s'attache au souvenir de ces actions génératrices de plaisir; la relation avec l'expérience initiale de passivité permet - souvent, seulement après des efforts conscients dont le sujet se souvient - de refouler ce reproche et de le remplacer par un symptôme primaire de défense. Scrupulosité, honte, méfiance de soi-même, sont les symptômes qui ouvrent la (...) période de santé apparente mais en fait de défense réussie. (p. 67) 


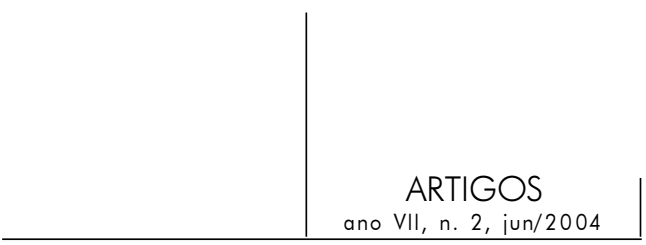

Nous pouvons entrevoir dans ce texte freudien et dans le manucrit $\mathrm{K}$ qui lui est contemporain, que Freud appréhende la honte en deux étapes qu'il conceptualise comme étant le déroulement typique de la névrose de contrainte, cette dernière se subdivisant en quatre phases. Premièrement, les symptômes primaires de défense tels que la pudeur, la scrupulosité, la moralité et les formations réactionnelles (étape précoce de constitution) vont permettre à l'enfant de se soustraire momentanément aux reproches qui l'assiègent. Deuxièmement, à l'étape du retour du refoulé, la honte se présente comme "un affect de contrainte" pour reprendre l'expression freudienne; il correspond à l'une des voies hypothétiques de l'affect initial de reproche si caractéristique de la culpabilité dans la névrose obsessionnelle. Freud écrit:

L'affect de reproche peut, grâce à une addition psychique, se transformer en n'importe quel autre affect de déplaisir; ceci accompli, plus rien ne s'oppose à ce que l'affect substitutif devienne conscient. Ainsi, le reproche (pour avoir accompli l'action sexuelle dans l'enfance) se transforme avec facilité en honte (si un autre pouvait l'apprendre), en angoisse hypocondriaque (crainte des conséquences corporelles nuisibles de l'action passible de reproche), en angoisse sociale (crainte du châtiment social du méfait), en angoisse religieuse, en délire d'observation (crainte de trahir à d'autres l'action commise), en angoisse de tentation (méfiance justifiée dans sa propre force de résistance morale), etc. (ibid., p. 69)

Ainsi s'ébauche le modèle opératoire freudien où la honte apparaît comme un dérivé de l'angoisse morale, impliquant une dimension défensive projective de la figure du surmoi sur le regard social. C'est précisément cette instance d'autoobservation qui est projetée dans le social à titre de défense secondaire contre l'assaut des auto-reproches obsessionnels; le sujet obsessionnel redoute avec la même acuité le jugement social, tout comme le jugement de son propre surmoi. Cette question de la honte chez Freud sera davantage développée dans le cadre de la névose de contrainte qu'à propos des autres névroses existantes; la question de l'analité et de la honte est ici étroitement intriquée.

C'est surtout avec la seconde topique que s'élabore le positionnement métapsychologique de la genèse du sentiment de honte. Dans "Psychologie des foules et analyse du moi", Freud (1921) reconnaît au Surmoi (Über-Ich) les fonctions suivantes: “... l'auto-observation, la conscience morale, la censure onirique et l'exercice de l'influence essentielle lors du refoulement” (p. 173). C'est le Surmoi qui donne le sentiment d'être surveillé et épié par une partie de soi-même, contribuant ainsi au caractère paranoïde du Moi. D'un point de vue métapsychologique, la honte est subordonnée à l'Idéal du Moi (Idéal-Ich) dans la mesure où elle est le reflet du conflit intra-psychique entre le Surmoi et le Moi, conflit entre les instances psychiques qui est déterminant pour le sujet. Dans "Le 


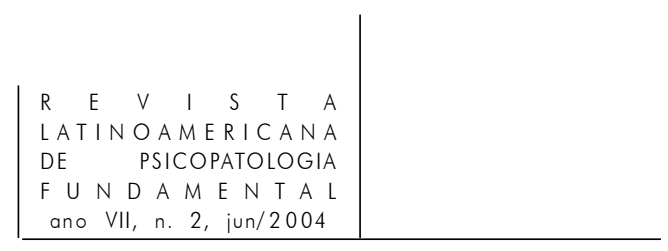

Moi et le Ça" (1923), l'auteur nous propose la formule suivante: "Tandis que le moi est essentiellement représentant du monde extérieur, de la réalité, le sur-moi se pose en face de lui comme mandataire du monde intérieur, du ça” (p. 249). C'est ainsi que l'éclosion des conflits entre l'instance moïque et l'Idéal du Moi vont inaugurer l'opposition entre monde extérieur et monde intérieur, entre réel et psychique dans un conflit inter-instances où les exigences du Surmoi despotique vont tyranniser le Moi, surévalué négativement par rapport à l'Idéal du Moi coercitif. Dans le vécu de honte se produirait ainsi une coalescence entre monde intérieur et monde extérieur: le Moi se trouve pris dans un double mouvement identificatoire entre d'une part le Surmoi qui juge et auquel il se réduit et d'autre part l'identification imaginaire de sujet "honteux" imputée au regard d'autrui. Une dialectique conflictuelle dynamique s'instaure entre le Surmoi et l'Idéal du Moi (comme figure surmoïque) qui s'effondre, pris dans les rets de cette instance "cruelle" qui le critique et par ailleurs point de mire du regard d'autrui, témoin de la fragmentation et du ratage de sa confirmation narcissique: "Le ça est totalement amoral, le moi s'efforce d'être moral, le sur-moi peut devenir hyper-moral et alors aussi cruel que seul le ça peut l'être" (ibid., p. 269) écrit l'auteur au sujet de la moralité et de la répression pulsionnelle.

Dans le même texte, Freud introduit la notion de Moi-Idéal (Ich-Idéal) mais il faut souligner que parfois, avec l'Idéal du Moi et le Surmoi, ces trois termes sont utilisés de manière synonymique dans l'œuvre freudienne, ne permettant que difficilement leur délimitation sémantique et même métapsychologique. En effet, l'auteur admet l'existence d'une différenciation, au sein du Moi, d'une instance qu'il nomme indifféremment Idéal du Moi ou Surmoi, issu du système perceptionconscience, comme ses propos en témoignent: “... l'idéal du moi ou sur-moi (est) la représentance de notre relation aux parents" (1921, p. 248). Le Surmoi est au centre de la question morale. Il a un rôle de juge et de censeur à l'égard du Moi. Il inhibe les actions du sujet, induit le remords, les scrupules et le repentir. Le MoiIdéal, lui, caractérise le moi réel, objet convoité des premières gratifications et satisfactions narcissiques. Et ce n'est pas dénué de nostalgie que le sujet aspire à retrouver ce Moi-Idéal conçu comme un idéal de complétude narcissique, édifié sur le modèle du narcissisme infantile: "Nous sommes au centre de tout désir de restaurer une toute-puissance à laquelle nous pensons et croyons avoir renoncé" (Jacobi, 1999). Tout ceci s'inscrit dans un rapprochement théorique que Freud opère entre ces deux instances du Moi-Idéal et de l'Idéal du Moi, leur attribuant conjointement les mêmes fonctions d'interdiction, de censure et d'idéalisation.

Pour Lacan, le moi idéal s'élabore à partir de l'image du corps propre dans le miroir, image qui constitue le support de l'identification primaire de l'enfant à l'autre semblable: "C'est cette image qui se fixe, moi idéal, du point où le sujet s'arrête comme idéal du moi. Le moi est dès lors fonction de maîtrise, jeu de 


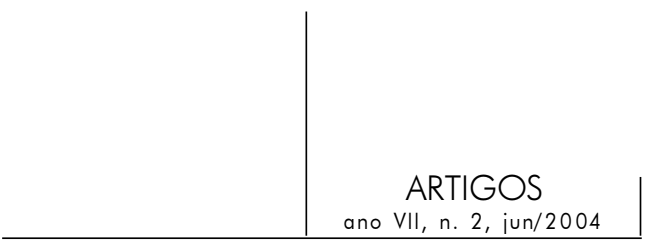

prestance, rivalité constituée" (p. 809). Ainsi, le sujet assume une certaine image de lui-même, enrichie des processus d'identification en perpétuelle mouvance, mais en aucun cas il ne peut se réduire à un champ exclusivement spéculaire de ce qu'il en est de son identification au miroir, car c'est toujours par la médiation de l'Autre, par le regard porté sur lui, que le sujet se perçoit et s'appréhende.

$\mathrm{Au}$ sens freudien, la culpabilité, contrairement à la honte, implique la transgression de la loi et concerne le Surmoi. Alors que la honte, en tant qu'éprouvé narcissique, est directement liée à l'Idéal du Moi. Elle implique une mise en défaut de soi, devant témoin. En ce sens, la honte a un caractère fondamentalement intersubjectif. Dans la terminologie freudienne, "Il se crée toujours une sensation de triomphe quand quelque chose dans le moi coïncide avec l'idéal du moi. De même, le sentiment de culpabilité (et le sentiment d'infériorité) peut être compris comme expression de la tension entre moi et idéal" (Freud, 1921, p. 201). Dans le vécu de honte, l'Idéal du Moi défaille en regard des énoncés impératifs surmoïques et en regard de l'Autre aussi, témoin de ces turpitudes et de sa déchéance. C'est ainsi que la honte manifeste la mise en défaut narcissique du sujet, en tant qu'il se repère et se détermine à partir de ce qu'il perçoit de lui-même dans le regard des autres. Lacan (1945) l'a exprimé dans une formule très éloquente: “... si dans cette course à la vérité, on n'est que seul, si l'on n'est tous, à toucher au vrai, aucun n'y touche pourtant sinon par les autres" (p. 212). En tant qu'affect, la honte ne nous laisse pas d'autre alternative que de reconnaître la primauté du regard des autres porté sur soi.

Dans une perspective classique, la métapsychologie de la honte dans l'œuvre freudienne rend compte de la différence du Surmoi comme instance critique et punitive à l'égard du Moi, et introduit la honte comme relation entre les différentes instances de la personnalité (Moi - Surmoi - Idéal du Moi) au sein de l'appareil psychique. Et si cette conceptualisation de la honte rend bien compte de la clinique, c'est aussi parce qu'elle est issue d'un ensemble théorique élaboré à partir des exigences de celle-ci, et non parce qu'elle témoigne d'une de ses manifestations particulières: "Surmoi, moi et ça sont donc les trois royaumes, régions, provinces en lesquels nous décomposons l'appareil psychique de la personne" écrit Freud en 1933 (p. 100-1). En ce sens, le modèle prototypique freudien rend compte de la honte dans son rapport exclusif au refoulement du sexuel, selon l'hypothèse phylogénétique, la découverte de la honte étant contemporaine de la découverte de la nudité.

Or, l'hypothèse de ce travail consiste à considérer que la clinique de la honte déborde cette conceptualisation pour se rapporter également au Moi-Idéal, référence à une complétude imaginaire qui constitue l'objet même de l'intérêt et de l'amour narcissique, en référence à une représentation de soi et de l'épreuve que représente le fait de se reconnaître imparfait, limité, manquant, c'est-à-dire 


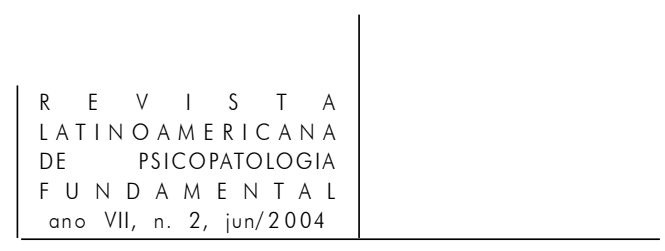

irrémédiablement soumis à l'épreuve de la castration. Le surgissement de la honte ne porterait donc pas seulement sur le sentiment de valeur et d'estime de soi mais aussi et surtout sur le sentiment d'identité, dont la honte en révèlerait les fissures et les ruptures. Dans cette perspective, la honte concerne directement le Moi-Idéal et manifeste la mise en échec, le ratage d'un idéal de toute-puissance narcissique. L'identification grandiose par laquelle le sujet essaie de reconquérir l'omnipotence infantile passée est brutalement mise à jour dans son achoppement même, ce que la honte révèle. Tout ceci vient en quelque sorte provoquer une réflexion quant à la conceptualisation de la honte dans le cadre de la seconde topique de l'appareil psychique et plus largement dans le champ de la psychopathologie freudienne. Dans l'élaboration de ce travail de recherche, la difficulté de rendre compte d'une métapsychologie de la honte - hypothèse théorique fondamentale - réside dans la prise en compte incontournable de cet objet qui est le regard de l'autre puisque la honte est d'emblée un sentiment social. Comme l'écrit Barazer (2000, p. 17), "En ce sens la honte pour être pensée nécessiterait une sorte de topique limite, à la limite de l'intra et de l'intersubjectif. Peut-être est-ce là une des difficultés à la penser méta-psychologiquement". Il conviendra de revenir sur cette conceptualisation.

Je vais maintenant vous présenter quelques séquences d'un suivi en consultation, celui d'une jeune femme, Madame K. âgée de 35 ans et de son enfant, Tony, âgé de quatre ans, né d'un viol. Les observations cliniques issues de ce suivi m'ont mises face aux expressions les plus archaïques de la honte - qui sont aussi, peut-être - les plus couramment admises; elles pourront à présent relancer un certain nombre de questionnements sur l'étroite correspondance entre traumatisme sexuel et sentiment de honte. Enfin, cette évocation clinique me permettra également de revenir sur l'hypothèse de travail indiquée au début de cet article et de montrer, au-delà, comment la prise en compte de l'éprouvé de la honte dans une écoute post-traumatique a des effets positifs sur la dynamique psychique du sujet.

\section{Étude de cas}

\section{Préambule}

La patiente dont j'exposerai quelques fragments d'histoire m'est adressée pour une situation assez dramatique de séquestration, de viol et de sévices. Les entretiens psychothérapeutiques avec Madame K. s'étendent sur une année et viennent soulever corrélativement l'ensemble des points suivants:

1. La confrontation du clinicien à des situations extrêmes, 


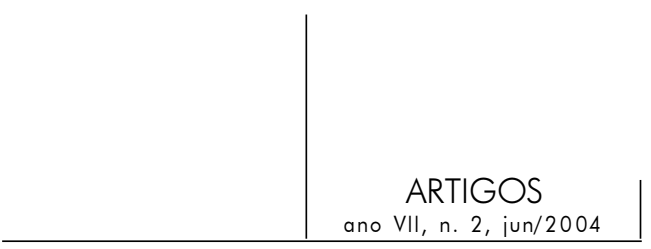

2. Le traitement psychothérapeutique de la honte, dans la succession des différentes temporalités psychiques et de leur étroite combinaison, passe par les trois temps suivants: l'apprivoisement de la honte, la mise en mots de la honte, l'affirmation et la reconstruction de l'identité.

\section{Éléments d'anamnèse}

Une dizaine de rendez-vous "manqués" se seront succédés avant que je ne puisse rencontrer Tony et sa mère. Jusque-là, Madame K. n'avait pas pu faire la démarche de venir en consultation.

Plusieurs signalements d'enfant en danger avaient été faits, notamment par l'une de ses sœurs, pour cause "de fuite de la mère avec son enfant et suspicion de mauvais traitements" mais à ce jour, aucune décision judiciaire et sociale n'avaient été mises en application. C'est précisément dans cette occurrence que furent transmis par l'Association pour la Protection de l'Enfance et de l'Adolescence des données anamnestiques précises. La symptomatologie présentée par l'enfant se déclinait en une succession de troubles énumérés ainsi:

Enfant coprophage qui présente une prévalence de:

- Troubles alimentaires,

- Troubles du comportement,

- Troubles du sommeil,

- Troubles du langage (enfant qui ne parle pas),

- Troubles liés à une suspicion de maltraitances maternelles.

Deux phrases dans le compte-rendu du médecin de Protection Maternelle et Infantile avaient tout particulièrement retenu mon attention:

C'est un enfant extrêmement inquiétant, décrit comme "non humanisé".

C'est la pire situation que nous n'ayons jamais rencontrée.

L'indication initiale de consultation avait pour motif la réalisation d'un bilan psychologique de cet enfant. Cette évaluation - demandée par le psychiatre devait être réalisée dans les plus brefs délais, compte tenu du caractère d'urgence de cette situation.

\section{Rencontres cliniques}

a) L'apprivoisement de la honte

Le premier entretien ne fut facile ni pour elle ni pour moi. Pour elle car j'étais soit jugée indifférente à ses demandes, soit incompétente à l'aider et à la comprendre. À une intervention de ma part comme quoi elle pouvait être aidée, 


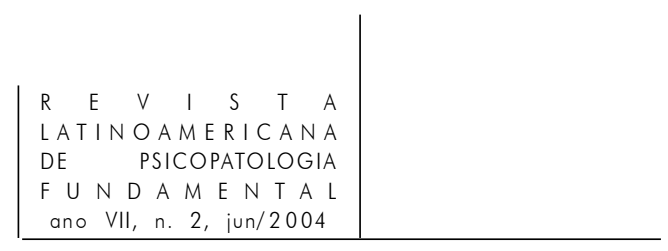

elle m'accusa d'avoir un optimisme mal placé dans mes efforts pour faire avancer la compréhension de son vécu très douloureux. Pour moi, cet entretien fut éprouvant, sans doute du fait de cette situation particulièrement difficile, celle de la rencontre d'une femme - qui a été séquestrée et violée - et de son enfant, né à la suite de ces actes barbares.

Tony est un petit garçon d'apparence chétive, qui émet simplement des sons pour exprimer ses demandes ou son mécontentement. Il manifeste une grande détresse lorsque sa mère lui pose un interdit. Les échanges entre la mère et son enfant sont empreints de violence, verbale et physique. Assise face à moi, la mère se tient entièrement de profil - face à la porte - et ne me regarde pas durant l'entretien. Elle affirme être venue consulter pour son fils, avec la ferme intention de "nous le laisser" dans le service. De manière itérative, elle me demande de le prendre en charge immédiatement à l'Hôpital de Jour: "Vous pouvez vous en occuper, vous ici". Je lui réponds alors que je ne suis pas en mesure de prendre une décision d'ordre médical. En réaction à cette réponse qu'elle estime déconvenue, elle exprime, non sans véhémence, son insatisfaction. Et alors qu'elle marmonne, je comprends qu'elle nous considère, psychologues, médecins comme étant "des incapables, juste bon à parler". Je commençais à croire moi aussi que je ne valais pas plus que ça, compte tenu de l'utilisation réduite qu'elle semblait faire de moi et surtout compte tenu du déroulement de cette consultation.

Après ces premiers échanges quelque peu tendus, elle commence à parler de son enfant, alors qu'il joue seul dans un coin de la pièce tout en émettant des petits cris. Cette mère put confier, à un moment donné: “Ce qui m'énerve, c'est que l'on me dise à l'école qu'il n'est pas normal. Tout se passe bien, sauf pour la parole... il ne lui manque que la parole". Cette évocation des difficultés scolaires et surtout, de manière implicite, du rejet dont Tony fait l'objet vont l'amener, à ma grande surprise, à faire une allusion au géniteur de l'enfant; la crudité de ces propos est choquante. Elle est alors prise d'une honte fulgurante - ses joues sont rouges - et elle commence à bégayer. Le sentiment de honte est manifeste, son malaise très visible: hésitation, silence, regard contrit, gêne colorent ses propos; il convient de noter toutefois que le mot "honte" n'est pas prononcé.

La défaillance sévère du moi, l'état de sidération et plus globalement la désorganisation psychique propres à l'état post-traumatique se manifesteront dans l'impossibilité, pour la patiente, de nommer son agresseur. Elle s'y essaiera une fois au cours de ce premier entretien et manifestera des expressions symptomatiques de honte, tels qu'une manifestation physiologique visible - le rougissement - et une difficulté langagière - le bégaiement - assortis de manifestations corporelles d'agitation et de nervosité. S'ensuivront un flot d'injures et d'insultes sur cet homme avant que Mme K., sollicitée par son enfant, ne s'interrompt pour aller lui chercher un jouet. Tony joue à lancer des jouets dans 


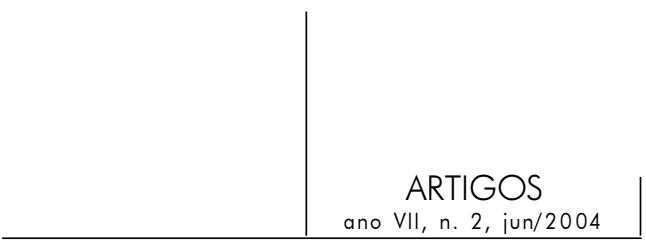

la pièce, et va faire mine régulièrement d'ouvrir la porte, tout en guettant la réaction de sa mère, qui ne se fait pas attendre. Le ton monte et les menaces de fessée se précisent.

Je ferais ici une brève digression pour rappeler que l'indication initiale de consultation avait pour motif la réalisation d'un bilan psychologique de cet enfant. Plus tard au cours de ce même entretien, je propose donc à la mère de nous laisser quelques instants seuls, l'enfant et moi. C'était une erreur. La mère réagit très mal à ma proposition. Contrariée, elle se lève et répète avec insistance que Tony n'acceptera pas de rester avec moi. Ses propos résonnent comme une injonction vis-à-vis de son enfant qui se montre réellement désemparé. Ce que j'entends très clairement, c'est qu'elle lui interdit de rester avec moi. Tony le comprend sans doute encore plus vite que moi. Il commence à pleurer alors que sa mère n'a pas encore franchi la porte. Durant une à deux minutes, j'essaierais en vain de l'apaiser. Mais sa détresse est telle que je me dirige rapidement vers la porte pour aller chercher sa mère. Au moment d'actionner la poignée de la porte, je découvre avec stupeur que la mère nous a enfermés. Elle retient la poignée de toutes ses forces. Je lui demande de me laisser ouvrir la porte. Au bout d'un moment, elle obtempère: “Je vous l'avais dit qu'il ne voudrait pas rester avec vous". À cet instant précis, je me découvre brutalement submergée par des sentiments d'angoisse tels que je n'arrivais plus à penser. Je me sentais à la fois sollicitée sans répit, mise à l'épreuve et en même temps réduite à l'impuissance.

Alors que cette première rencontre s'achève, la patiente dira, toujours dans l'évitement de la rencontre de nos regards: "J'ai trop honte pour vous parler de ce qui m'est arrivé...”. Elle affirme aussi, alors que je note le prochain rendezvous, qu'elle ne reviendra pas. Je lui dis alors à quel point il me paraît important, pour elle-même et son enfant, de ne pas rester sans accompagnement psychologique, éducatif et social. Laconique, elle rétorquera: “Donnez-le toujours ce rendez-vous, vous verrez bien si je viens ou pas".

Après les avoir raccompagnés, je retourne dans mon bureau et après m'être assise, je me sens aux prises avec une pensée confuse qui se dérobe. J'ai le sentiment, pour ne pas dire la certitude, de ne pas avoir pu accueillir cette mère et son enfant. Dans les jours qui suivirent, je fis des cauchemars: l'image la plus impressionnante fut la nette apparition de corps de femmes et de fœtus monstrueux; lié à cela, le spectre terrifiant d'une grossesse issue d'un viol. C'est dire à quel point, face à des situations extrêmes, le clinicien peut être envahi de mouvements émotionnels et fantasmatiques divers. Ces cauchemars étaient le fruit d'une actualisation transféro-contre-transférentielle où avaient été mêlés répulsion et effroi; bouleversée par cet entretien, habitée par la confusion, je réalisais que le trauma sexuel interroge le clinicien au plus intime de son contre-transfert, ici sous forme d'émois tels que je viens de l'évoquer. Je citerais ici Lévy (2000) 


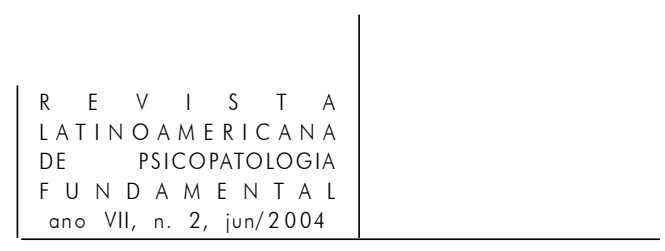

lorsqu'il écrit, au sujet des situations traumatiques extrêmes, que le moment contre-transférentiel est un "... moment à ne pas rater dans ce qui risque à tout instant pour l'analyste de l'emporter dans des réactions de dépit et de rejet, face à l'échec de toute interprétation, au sentiment d'un sur-place..." (p. 60) autrement dit d'une impasse face à la désymbolisation traumatique et à ses ravages au cœur même de la subjectivité.

La mère me téléphone quelques jours plus tard: "Je 'pète les plombs', je n'en peux plus!". Je lui suggère de venir en parler. Elle ne viendra pas au rendezvous fixé. Plusieurs appels seront nécessaires pour pouvoir se rencontrer de nouveau. En ce qui me concerne, ce temps-là aura été bénéfique. J'ai dépassé les images terrifiantes qui ont entouré la conception de Tony, cet enfant en si grande souffrance, comme sa mère.

b) La mise en mots de la honte

Durant une première période, Mme K. pleure beaucoup durant les entretiens. Un jour, elle commence à évoquer les faits traumatiques survenus dans sa vie, à savoir la séquestration suivie de violences sexuelles dont elle a été victime, et surtout sa culpabilité liée au fait de ne pas avoir pu s'opposer à ces actes: "Je m'en veux, je me dégoûte moi-même" dit-elle. Murée dans une culpabilité massive, ces paroles ne semblent à aucun moment se soulager d'être dites, ce qui me fait penser que le problème est à situer ailleurs, c'est-à-dire du côté de la honte de ce viol. Curieusement, son discours apparaît dénué d'affects et d'émotions, en total décalage avec la gravité de son contenu. Selon l'analyse de Barazer (2000), les sujets victimes de traumatismes sexuels peuvent “... 'donner en pâture' à l'analyste le plus intime d'eux-même mais comme s'ils l'expulsaient comme déchet et non se l'appropriaient par la parole" (p. 11). Les traces mnésiques laissées par ces événements apparaissaient ainsi comme des fragments épars, revenant sans cesse au cours des entretiens mais comme désaffectés, aseptisés. Les faits étaient imprégnés d'un style narratif très particulier, descriptif, factuel.

De la récurrence des auto-reproches va progressivement se déployer, dans l'espace transférentiel, un flot d'injures à l'encontre du violeur. Ce qui importe, c'est qu'elle puisse enfin déposer - dans le site psychothérapique - l'outrage extrême d'avoir été contrainte à des rapports sexuels. Un jour, elle dit: "J'aimerais être moi, enlever toutes ces choses dégoûtantes de mon esprit...".

De "dégoût", de "choses dégoûtantes", il en sera beaucoup question durant ces premières semaines, mais le mot "honte" ne sera jamais énoncé. Autrement dit, elle ne peut mettre en circulation cette honte dans le transfert qu' à la condition de la dire sous une forme travestie, le dégoût venant la désigner et exprimer directement le rapport au corps, "le dégoût de ne pas avoir pu empêcher cela". 


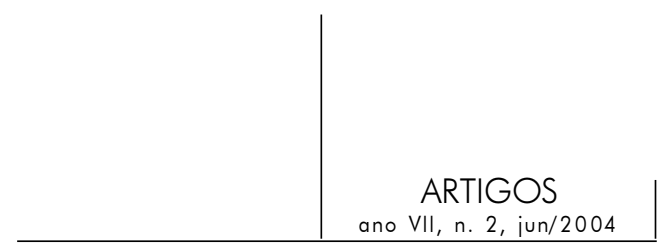

Enfin, à partir du moment où elle va commencer à se (ré)approprier ces éléments de son histoire et surtout les faits traumatiques, d'intenses manifestations dépressives et une succession d'interruptions des entretiens vont révéler toute la difficulté à sortir de la barbarie dans laquelle l'ont immergée ces événements. C'est dire aussi que dans la dynamique transféro-contre-transférentielle, la factualité événementielle initiale commençait à advenir psychiquement comme souvenirs du passé, vérité historique. Mais, et de façon plus large, ces vagues réminiscences constituaient un support sur lequel pouvait éclore une trace, une ébauche de représentation de l'(im)pensable traumatique. Alors que la patiente avait évoqué, au début de nos rencontres, un vécu de culpabilité lié aux violences sexuelles subies et à son impuissance, c'est un vécu de honte qui va bientôt occuper les entretiens.

Trois mois séparent l'entretien qui suit de notre première rencontre. Je reprendrai ici à ce point précis un passage extrait de cet entretien, qui met en relief un certain nombre de questionnements ainsi que les prémices de l'accès à une expérience subjective. Madame K. est assise en face de moi mais se tient toujours de profil, manifestant avec la même acuité l'évitement de la rencontre de nos regards. Elle a le visage tendu, se triture les mains, semble embarrassée. Elle finit par dire, le regard contrit: "Les images du viol se déroulent comme un film permanent... je n'arrive plus à laisser le passé derrière moi... il m'obsède”. À cette évocation, son regard se brouille et des larmes coulent de ses yeux où on pouvait lire un grand désarroi. Non sans une grande confusion émotionnelle, elle parviendra juste après à confier: "J'ai honte de ce qu'il m'a fait". C'est à partir de là que la patiente commencera à parler d'elle, en livrant, à demi-mot, les fragments traumatiques de son histoire. Effraction du corps et de l'être, le viol est une atteinte irréversible au désir, au féminin et à la sexualité.

À des bribes de phrases succéderont des silences ponctués de soupirs. Je note qu'au fur et à mesure où elle va mettre en récit les actes barbares subis c'est-à-dire la séquestration et les violences sexuelles répétées, des modifications de la posture corporelle se dessineront progressivement mais nettement. En effet, la patiente se redresse peu à peu sur sa chaise et se tourne à présent vers moi. Elle ne se tient plus de profil mais de face et commencera d'abord par me jeter des regards furtifs.

c) L'affirmation et la reconstruction de l'identité

C'est à partir du moment où elle aura pu confier être victime de honte, avoir honte de ce viol, que la patiente va commencer à détailler les conditions de la séquestration ainsi que les sévices sexuels subis. Autant dire que c'est à partir de la nomination de la honte qu'elle va pouvoir aborder le contenu des scènes 


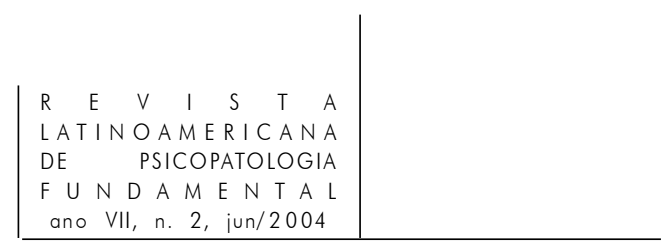

traumatiques. Dans les semaines qui suivront, elle décrira, de manière itérative, les violences subies, répétition qui manifeste une tentative d'élaboration psychique et de (ré)appropriation subjective. Après le dépassement de "la honte de la honte", la patiente essaie de dépasser et de métaboliser les souvenirs des évènements traumatiques qui envahissent son présent et qui prennent figures d'obsessions. Enfermée dans ses souvenirs inoubliables, elle se demande comment supporter cette réalité ressentie comme intolérable, celle de sa propre histoire. En même temps, en me parlant de ces images qui s'imposent à elle en permanence, elle révèle que quelque chose ne peut et ne pourra jamais se transmettre, à savoir une expérience dont les mots s'avèrent impuissants à la soutenir, la représenter, l'expliquer. Et c'est bien de l'horreur du trauma dont il s'agit. C'est cela aussi qui est informulable. Il convient de noter qu'à l'émergence du trauma psychique apparaîtront toujours étroitement intriquées les injures sur l'agresseur, violeur, bourreau, comme tentative de s'arracher à l'abject en tant qu'objet de la pulsion de l'autre. Mais plus. Comme tentative de transformation des traces en pensée, des reviviscences en remémoration. "Le salaud, c'est lui". Je rejoindrais ici Jacobi (2000) lorsqu'il affirme que la plainte qui désigne l'agresseur constitue “... un indice décisif dans l'élaboration psychique consécutive à une violence sexuelle" comme prémices d'accès à la possibilité d'une restauration narcissique, lourdement grevée par le traumatisme. Reconnaître la cruauté, l'abomination de l'objet constitue une tentative désespérée, et ultérieurement salvatrice, de reconquête et de (ré)appropriation de sa subjectivité, anéantie par l'horreur traumatique.

Un an s'est écoulé depuis le début du suivi. Ce jour-là, Tony est présent avec sa mère à l'entretien; très affairé auprès des jouets qu'il a déniché dans la malle, il vient plus tard nous solliciter pour participer à ses jeux. Il fait des allers et venues entre sa mère et moi, émettant des petit cris et semblant vouloir nous impliquer à son activité favorite, lancer une balle contre un mur: "À la maison, c'est pareil... il peut faire ça pendant des heures" souligne la mère. Tony vient plus tard sur les genoux de sa mère réclamer de la tendresse. Madame K. sourit. C'est la première fois que je la vois esquisser un sourire. Tous deux sont dans un échange de tendresse et d'affection. La mère me regarde à présent et sollicite mon regard. Au moment de nous séparer et alors que tous deux se dirigent vers la porte, Tony se retourne et revient vers moi pour m'embrasser. La mère est surprise de l'initiative de son enfant. Elle dira: "Il est venu vers vous, il vous a adopté. Ce n'est pas un monstre... pour vous, il n'est pas monstrueux...”. 


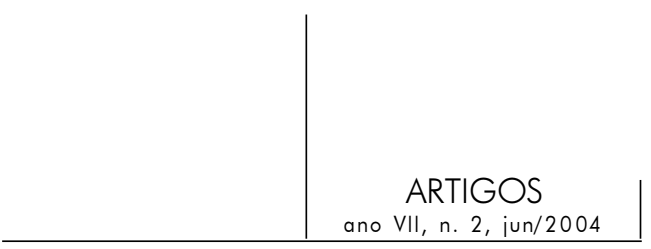

\section{De l'indicible du trauma sexuel à l'émergence de la honte dans le transfert}

L'analyse de ce fragment clinique me permet à présent de revenir sur l'affirmation indiquée au début de cet article, à savoir que l'une des fonctions de la honte, c'est de permettre au sujet de s'éprouver comme tel. Dans ce parcours à partir, autour de la honte, dans cette approche du champ de la honte, vont être développés à présent les trois temps subjectifs aptes à conceptualiser le traitement psychothérapeutique de la honte:

- Dans un premier temps, "la honte de la honte" ne permet pas au sujet d'en parler, dans la mesure où c'est la honte elle-même qui interdit sa propre expression: c'est le temps de l'inhibition.

- Dans un deuxième temps, après avoir apprivoisé la honte dans un cadre approprié à l'accueillir, la mise en mots de la honte par le sujet sera effective: c'est le temps du regard.

- L'expression et la reconnaissance de la honte par le sujet vont lui permettre l'accès à une expérience subjective: c'est le temps de l'identité.

a) Le premier temps: l'inhibition

D'un point de vue métapsychologique, l'inhibition correspond à un processus physiologique actif et réversible, de suspension temporaire d'une fonction par une autre. Le mouvement rétrograde imprimé au sujet par l'importance de l'inhibition s'étend à la sphère somatique et psychique. Les perturbations fonctionnelles se définissent de façon négative par le fait qu'une activité ne peut avoir lieu: "Ces perturbations fonctionnelles, expression d'une limitation du moi, constituent ce que l'on appelle des 'inhibitions"" (Chemama, 1993, p. 147). Le Moi renonce à certaines fonctions pour ne pas avoir à entreprendre un nouveau refoulement, pour ne pas entrer en conflit avec le ça. Mais, écrit Freud (1926), "Relié intimement au ça lui-même, il ne peut écarter défensivement le danger de pulsion qu'en restreignant sa propre organisation et en s'accommodant de la formation de symptôme comme substitut du préjudice porté par lui à la pulsion" (p. 68). Le Moi, lorsqu'il est confronté à une épreuve psychique difficile, ici une énorme répression d'affect, connaît un appauvrissement en énergie qui manifeste la restriction fonctionnelle moïque et que caractérise l'expression de l'inhibition.

L'inhibition comme manifestation symptomatique de la honte était manifeste dès le premier entretien. Cette inhibition était à la mesure de la forte mobilisation défensive sous-jacente. Mme K. exprimait le désir d'une rencontre mais la surenchère défensive visible témoignait de la forte angoisse mobilisée dans cette 


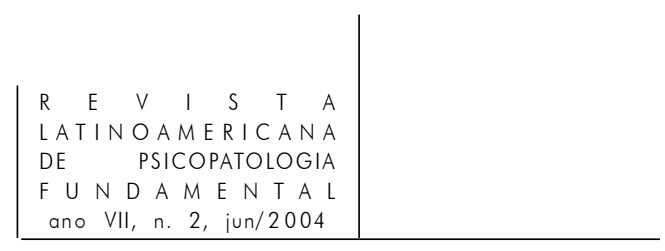

occurrence. L'évitement de la rencontre de nos regards traduisait à mon sens la dimension de "honte de la honte" mais d'agressivité aussi. La manifestation corporelle d'évitement (elle se tenait de profil) rendait nos échanges difficiles. Dans la relation transférentielle, les conditions de notre rencontre étaient parsemées de contraintes multiples. Se conjuguaient les injonctions de l'éducatrice spécialisée de L'A.P.E.A. concernant la rencontre de la psychologue dans une perspective de bilan psychologique, l'obligation de soins et les menaces de placement de l'enfant en famille d'accueil en cas de non observance de ces indications.

Lorsque Mme K. essaie de nommer son agresseur, la manifestation physiologique de rougissement et le bégaiement comme expression de la confusion émotionnelle attestent de cette (ré)actualisation de la honte dans la relation transférentielle. De rester détournée physiquement du clinicien traduisait une volonté manifeste de se soustraire de son regard et du même coup de s'en protéger. Comme si le regard tiers, suite à l'énonciation des faits, devenait momentanément insoutenable. Mais on peut formuler une autre hypothèse interprétative: ce détournement peut traduire le moment durant lequel le sujet est volontairement tourné vers lui-même, contemplant sa propre réalité intérieure suite à la révélation des faits. Par ailleurs, à son corps défendant, le sujet pris d'un accès de honte trahit - par des manifestations corporelles - son malaise et son désarroi, ce que Tisseron (1992) souligne en ces termes: "Le sentiment vécu de la honte, parce qu'il s'accompagne de postures corporelles, de mimiques ou d'intonations particulières, informe l'environnement" (p. 179). Ces manifestations corporelles, qui ont une vocation de défense, sont autant d'éléments qui mettent le clinicien en alerte sur un vécu potentiel de honte chez le sujet. Sous l'effet de la honte, le corps est momentanément sans force et sans énergie. La honte donne (du) corps dans le sens où elle donne une réalité, une forme de consistance se matérialisant par des symptômes corporels: le sujet devient rouge, transpire, ressent des sueurs profuses et des palpitations cardiaques, se tripote nerveusement les mains et cherche à se cacher du regard d'autrui. Bref, ces expressions symptomatiques montrent que la corporéité (matérialité) est fortement sollicitée et concernée dans l'expérience de honte. La honte se déploie littéralement au sein de l'espace corporel; dans son effusion pulsionnelle, elle se condense et se meut à la fois dans le psychisme et le corps; elle concerne simultanément la sphère somatique et psychique.

Un des effets physiologiques de la honte, outre l'accélération des pulsations cardiaques et les phénomènes éventuellement neuro-végétatifs, est le rougissement comme contremarque de l'afflux pulsionnel sous-jacent. Le rouge de la face est peut-être le signe le plus invalidant pour le sujet, car il ne peut être ni empêché ni à fortiori camouflé. Le rougissement se voit et cette évidence est cuisante pour 


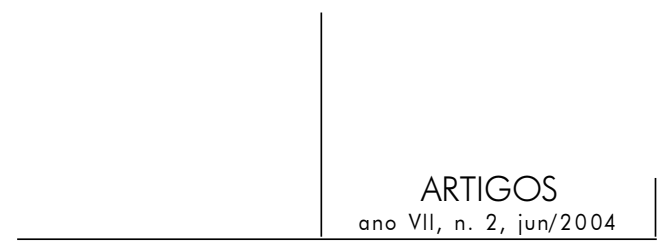

le sujet qui s'en sent doublement humilié: à la honte de rougir ainsi se conjugue la honte d'être livré à autrui, impuissant et dans une position d'infériorité dans le fait de ne pas avoir pu contrôler son émoi. Dans sa crudité la plus absolue, la honte met le sujet à nu, le visage en portant le témoignage et le (dé)voilement rougeâtre en révélant l'assaut pulsionnel et l'émoi narcissique sous-jacents. Bref, ce qui était initialement caché ne l'est plus et l'effet corporel visible de la honte vient mettre à jour, dans une décharge fulgurante, ce qu'il en est de l'intériorité du sujet - effet de transparence - pris sous le regard d'autrui dans sa vulnérabilité la plus absolue. Dans l'ensemble de ces manifestations symptomatiques, c'est la honte dans sa matérialité corporelle qui est mise en relief. Nous pouvons dire qu'en tant que phénomène, elle "utilise" le corps pour pouvoir se dire et s'exprimer.

Émergence de cet affect sur fond de déplaisir, la honte vient parler pour la patiente et témoigner de cette horreur du réel qui l'a brutalement arrachée à sa dignité de femme et de sujet. L'état de confusion honteuse et le bégaiement manifesté témoignent de cette impossibilité actuelle à inscrire le traumatisme dans le registre des mots. Je citerais ici Gori (2000) lorsqu'il dit, au sujet du traumatisme: “Dans ces conditions lorsque le maillage psychique n'a pas été réalisé dans les traces du rêve, du transfert ou d'une parole pleine, ne subsistent que le réel et l'affect d'effroi qui l'accompagne". L'impossibilité de nommer le nom du violeur renvoie fondamentalement à l'impossibilité de nommer l'irruption traumatique et l'horreur du viol comme épreuve innommable. La honte manifestée témoigne de cette mise à nu de l'effet traumatique, trauma exclu de toute symbolisation, sans médiation de mots. Le bégaiement en tant que manifestation langagière de la honte exprime l'état de confusion psychique propre à la réminiscence de la scène du viol et de la honte éprouvée. Mais plus. Elle traduit, au-delà, l'enfermement de la patiente dans la survie de cet événement inoubliable et dans cette épreuve de déréliction incommensurable de ce qu'elle ne peut encore mettre en mots. Ferenczi (1932, p. 100) écrit à ce sujet: "La confusion correspond au moment situé entre la surprise et la nouvelle adaptation". L'abord de l'événement traumatique surviendra ici, dans l'après-coup, fragment par fragment, dans la confusion et le tremblement des mots; ce qui est vecteur de terreur, c'est de formuler l'énoncé et de l'entendre: "L'effet d'une parole ne tient pas à son expulsion, mais à sa capacité d'inscription pour celui qui peut s'entendre la dire" (Jacobi, 2000). La sidération du trauma fige le temps, le pétrifie et assujetit le sujet au bégaiement qui atteste d'un achoppement de l'inscription de l'horreur dans le registre des mots. Le sentiment de honte, très marqué, était perceptible à travers le bégaiement et la rougeur évidente. Ce que Assoun (1995) écrit ici peut nous éclairer sur cette dialectique: "Le sentiment social tient au regard - par la honte - et à la voix - par la culpabilité. Double appartenance du sujet au qu'en verra- 


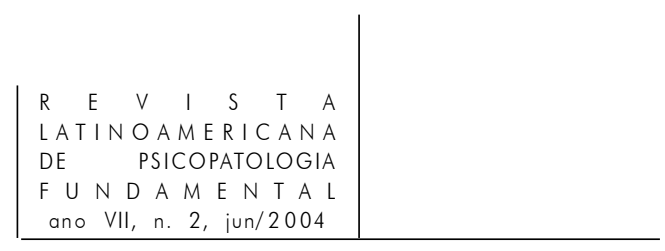

t-on (de son objet) et du qu'en dira-t-on (de soi)" (p. 32). Comment faire partager cet indicible, cet impensable de la souffrance au cœur même de la subjectivité? La situation traumatique est paradoxale puisqu'elle génère une souffrance à la fois impensable, non "historicisée" et en même temps incontournable, inévitable. La dimension d'effroi et d'horreur des réminiscences de cet événement traumatique qui a fait brutalement effraction dans la vie psychique du sujet, le fige et l'hypostasie à cette expérience désubjectivante; elle l'amène aussi, dans le spectre du souvenir traumatique et de sa force de contrainte et de répétition, à reproduire, dans l'actuel de la thérapie, l'impensable de cette situation, le non-élaborable du souvenir qui lui est attaché.

Ferenczi (1932), qui a joué un rôle essentiel dans la mise en place de repères analytiques propres à permettre de comprendre la notion de traumatisme, explique que suite "... à la découverte et la reconstruction du trauma supposé, suit une série quasi infinie de répétitions dans les séances d'analyse, avec toutes les explosions d'affects inimaginables" (p. 162) comme des réactions de colère, de révolte, d'effroi, de terreur, d'angoisse, de vengeance. Or, et c'est là un point essentiel, le viol imprime chez la victime des sentiments de culpabilité et de honte, culpabilité de ne pas avoir pu empêcher cela, honte "d'avoir fait cela" comme le confiera la patiente en cours d'entretien. La culpabilité est alimentée par les ruminations incessantes qui prennent allure d'obsessions, celles de ne pas avoir pu éviter le viol, celle de l'effraction et de la passivité manifestée. Précipité dans l'horreur d'une épreuve innommable, le sujet violé se sent coupable et honteux de ce dont il est victime. Culpabilité d'avoir commis une faute, d'avoir transgressé, manquement dans le fait de ne pas avoir pu empêcher ce viol. Honte de la souillure sexuelle, de la pénétration forcée, honte d'avoir été l'objet de la jouissance de l'autre. Comme le dit Jacobi (2000), le sujet victime de violences sexuelles éprouve la "honte d'être devenu déchet, honte d'être déchet par la déchéance infligée". En tant que femme violée, honte d'avoir été le témoin forcé de cette jouissance prise par l'autre dans une proximité sexuelle et corporelle insoutenables et subies. Effraction indélébile de l'être même, dans son psychisme et sa chair, le viol est une atteinte à la sexualité, à la féminité, à l'identité.

b) Le deuxième temps: la mise en mots de l'événement traumatique

La réification de sujet victime d'actes barbares assignait à la patiente une place particulière, celle de femme violée, de victime. Comme l'a noté Ferenczi (1932) au sujet de l'expérience traumatique, "Une personne ainsi devenue paralysée dans son activité de pensée doit être incitée au travail de pensée en renouant avec les images mnésiques vagues, faibles - ou avec des fragments de celle-ci" (p. 72). La verbalisation de la situation traumatique, la remise en 


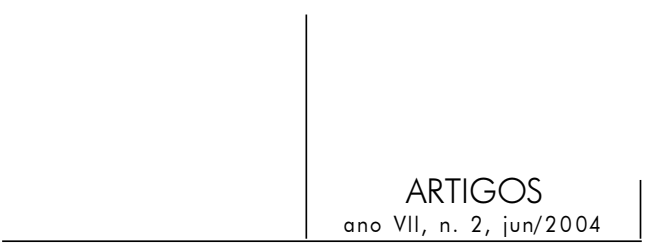

circulation de cet indicible jusque là hypostasié par la honte à dire et honte de la honte, témoignent, dans l'espace transférentiel, d'une réintroduction du traumatisme et de la honte à la temporalité de la thérapie. À la mise en mots des violences sexuelles va se déployer une série de manifestations corporelles et physiques qui attestent de l'émergence d'une (ré)appropriation subjective par la parole. Le dépassement de "la honte de la honte" permet de nommer la barbarie et d'ouvrir la voie psychique à une ébauche d'élaboration. La patiente va déployer, dans la pénombre des mots et des silences, les bribes de la scène traumatique, la séquestration, les violences physiques, sexuelles, dans une tentative de liaison des impressions sensorielles, émotions et représentations. L'accès à une expérience subjective commence ici, dans la réintroduction du traumatisme dans la temporalité de la thérapie, dans sa restitution au sein de l'histoire individuelle et de son économie psychique.

Dans la dynamique intersubjective, la mise en mots du trauma par la patiente va entraîner une modification très perceptible de sa posture corporelle. En effet, au fur et à mesure qu'elle évoque, à demi-mot, l'événement traumatique, elle va progressivement modifier sa posture physique et corporelle. De profil en début d'entretien, elle va se redresser sur sa chaise et se tenir face à moi à la fin de la séance. Nous sommes alors dans un véritable échange des regards.

La mise en mots du traumatisme contenue dans la révélation " $j$ 'ai été violée" va permettre au sujet de se faire reconnaître comme sujet victime, sujet violenté, mais sujet d'abord. Le passage par la parole va briser la morbidité silencieuse, la loi du silence et la dimension d'asymbolisation. Quelque chose de la honte transpirait dans les manifestations corporelles, émotionnelles, posturales et physiologiques notées au cours des entretiens. En particulier, l'évitement de la rencontre de nos regards traduisait de manière très explicite la dimension de honte comme cause de cet évitement. Cette évocation du traumatisme qui s'effectue dans la honte n'est pas sans rappeler la formule de Barazer (2000), qui situe la honte “... au carrefour de ce triple processus d'exposition, de dépossession et de déchéance de soi face à l'autre” (p. 9). Dans ce sens, l'exposition de soi concerne le sujet ayant été crûment l'objet d'attaques portant sur son intégrité physique, somatique et sexuelle, la dépossession de soi concerne essentiellement le corps propre, brutalement confronté à l'irruption et la violence pulsionnelles de l'agresseur qui s'approprie le corps de sa victime comme objet de jouissance; de cette précipitation dans l'horreur, de cet impensable traumatique, la déchéance ressentie en porte le cruel témoignage: infigurable de la douleur, présence inoubliable et non-élaborable de la scène traumatique et impensable de cette situation psychique, impartageable lorsque le sujet est brutalement ravalé au rang de chose, d'objet sexuel voire de déchet abject. Ce tryptique d'exposition, de dépossession et de déchéance de soi rejette le sujet dans une incommensurable 


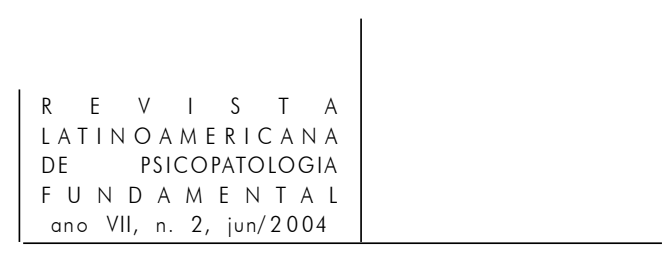

solitude, dans une unicité honteuse, indécente, dépouillée de toute capacité de subjectivation et de symbolisation.

c) Le troisième temps: le processus de (ré)appropriation subjective de l'événement traumatique par le sujet

Madame K. est venue initialement consulter dans un état de grande détresse et de désarroi, d'abord affectés à son fils, puis ultérieurement reconnus pour ellemême. À la lumière de cette vignette clinique, nous voyons comment, dans le cadre d'une consultation thérapeutique, la reconnaissance de l'éprouvé de la honte par la patiente a fonctionné comme (ré)amorçage d'un lien dans une situation d'isolement et de souffrance. Se reconnaître et se faire reconnaître sujet honteux, c'est déjà s'affirmer sujet. Comme le dit Miollan (1998, p. 164), "pouvoir montrer sa honte, c'est obtenir un regard de l'autre qui servira de contenant provisoire". Ainsi, cette demande de (ré)instauration de liens se nourrit et se maintient dans l'échange des regards, regard ayant à la fois une fonction de contenant et une dimension sociale.

L'hypothèse interprétative présentée trouverait validation dans son orientation transférentielle. La honte suspend la parole singulière du sujet, dans la mesure où c'est la honte elle-même qui interdit sa propre expression: "J'ai trop honte pour vous parler de ce qui m'est arrivé...” avait indiqué la patiente lors de notre première rencontre. Par conséquent, la mise en mots de l'éprouvé de la honte fut impossible dans un premier temps, du fait d'une part de la forte honte qui avait accompagné le traumatisme sexuel - qu'elle abordera plus tard - et d'autre part du fait du redoublement de cette honte à la remémoration et/ou la tentative de verbalisation de ces souvenirs. C'est dire que la honte peut envahir l'ensemble de la personnalité et s'opposer ainsi à sa mise en mots. Or, refuser de parler de sa honte, c'est se vivre comme prisonnier de la situation qui l'a engendrée. C'est aussi la renforcer, voire la pérenniser.

Cette évocation clinique amène à constater que ce n'est qu'après un apprivoisement de la honte dans un cadre approprié à l'accueillir, que la patiente est passée de l'expression simultanée de "ne pas pouvoir guérir de sa honte" et de "ne plus pouvoir se regarder en face" à une verbalisation de plus en plus détaillée de son vécu de honte, qui atteste d'une première mise à distance. Elle passe, dans ce travail, d'une adjectivation à une subjectivation. Elle est sujet de son discours et ce discours fait disparaître sa honte en tant que caractéristique de son image. Dans une perspective métapsychologique, ce passage qui traduit un mouvement à la fois intrapsychique et inter-relationnel illustre la dialectique du passage d'un fonctionnement essentiellement moïque, du côté du spéculaire et de l'identification imaginaire, à une ébauche de subjectivation, à une expérience subjective. 


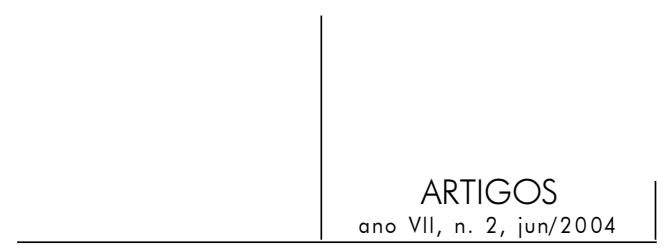

C'est dire que la prise en compte de l'éprouvé de la honte dans une écoute post-traumatique a des effets positifs sur la dynamique psychique du sujet. Lorsque l'expression de la honte devient effective, c'est d'abord à l'adresse du thérapeute: il s'agit pour le patient de verbaliser son vécu de honte, en lui donnant un sens. C'est-à-dire que lorsque le patient met en discours sa honte, celle-ci devient, dès lors, un objet de discours, un concept mentalisé, ce qui va permettre au sujet de prendre de la distance. Lorsqu'il dit " $j$ 'ai honte”, il a déjà conscience de sa honte et il change le statut de la honte qui, d'attribut, devient objet. Il affirme, dans cette mise en mots, qu'il espère s'en libérer. Tant que cette honte n'est pas dite, elle envahit le sujet et "colle" littéralement à son moi.

Dans le processus thérapeutique, la honte mise en mots par le patient et/ou éventuellement introduite par le thérapeute va lui permettre de la repenser et de l'historiciser, c'est-à-dire de la réintroduire à la temporalité, dans l'actuel, ce qui la rendra "travaillable". C'est une condition essentielle pour qu'il y ait effet thérapeutique, c'est-à-dire dégagement de la situation vécue avec honte. Pour le sujet, il s'agit de témoigner voire de dénoncer les situations de honte auxquelles il a été confronté; pour le clinicien, il s'agit de mettre le patient en face de ce qui ne peut être gardé en soi... et de l'aider à y faire face. Ainsi, la patiente confiera, lors de notre dernière rencontre:

J'ai longtemps refusé de parler, à cause d'une certaine honte de ce que j'ai vécu, à cause surtout d'une certaine honte de moi-même. Aujourd'hui, je parle de ma honte et je me sens mieux... disons qu'elle n'occupe plus le devant de la scène. Je peux désormais me regarder en face dans le miroir, me maquiller et me convaincre que je ne suis pas un monstre mais un être humain.

\section{Conclusion}

L'intention du cheminement clinique entrepris à travers cette réflexion sur une psychopathologie de la honte serait de témoigner de la trame dialectique honte \& trauma sexuel.

La rencontre de Madame K., femme violée et séquestrée, à jamais traumatisée, et de son enfant né à la suite de ces actes barbares, représente probablement la situation la plus difficile à laquelle j'ai été confrontée. La forte mobilisation des affects, la communication verbale rendue particulièrement difficile ont largement contribué à la difficulté d'aménager le cadre thérapeutique. Dans ce domaine de la maltraitance, les manifestations affectives et émotionnelles des victimes témoignent des répercussions traumatiques pathologiques. Comment le clinicien, face à une telle situation, assume-t-il la confrontation aux atteintes 


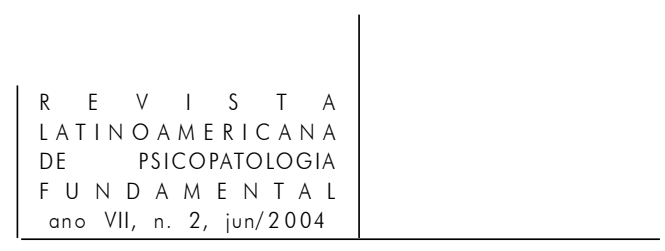

simultanées de l'enfant et de l'adulte? Comment peut-il répondre, de sa place, à de tels processus destructifs coupés de toute symbolisation, au point de mettre en défaut ce qui le spécifie, lui, clinicien? Comment gérer l'interrelation entre la mère et son enfant lorsque celle-ci est marquée, comme c'est le cas ici, d'une agressivité majeure?

Dans la prise en compte concrète de l'expression symptomatique, la mère apparaissait très fragile et fragilisée, voire même défiante à l'égard du soin psychique. Jouer avec l'enfant qui me sollicitait régulièrement en présence de sa mère a introduit un espace tiers, médiatisé, support potentiel de l'identification et de la mobilisation maternelles. Le support thérapeutique a permis d'aider la mère en difficulté à percevoir son enfant comme étant doué d'un potentiel de communication, d'expression et d'émotions personnelles. La circularité des échanges émotionnels a favorisé chez la mère des processus d'identification au thérapeute mais aussi à son enfant. ${ }^{7}$

La rencontre d'une mère et de son enfant nous montre qu'une alliance ne peut se construire et s'élaborer que dans un accueil et un "apprivoisement" des deux protagonistes, dont il faudra sans cesse stabiliser les excès et les manques après analyse des processus en jeu. La présence de l'enfant dans la consultation induit un climat émotionnel particulier, où les affects et les sentiments du clinicien sont fortement mobilisés, en écho de ceux de la mère. En ce qui concerne Mme K., l'accueil de l'histoire maternelle dans une écoute et une formulation a permis l'ébauche et le tissage d'un lien thérapeutique.

Chaque prise en charge est une rencontre singulière et dans l'établissement de la communication émotionnelle, le corps du clinicien, mobilisé dans sa sensorialité, son affectivité et sa pensée, participe du support thérapeutique. Enfin, la prise en considération des symptômes de l'enfant sert de point d'ancrage pour observer, identifier et verbaliser ce qui se joue dans la relation intersubjective maternelle.

Le recours à la consultation thérapeutique a permis la verbalisation, la mise en paroles du traumatisme subi; cette énonciation du viol subi, de la violence liée à la séquestration, bref, la révélation de ces actes exprime une tentative de subjectivation, de (ré)appropriation de sa subjectivité. La honte a une valeur structurante dans le maintien et la reconstruction subjective du sujet. Reconnaître sa honte permet de retrouver l'aptitude à penser et à symboliser. La honte témoigne que même réduit à être l'instrument de jouissance de l'autre dans une violence

7. Le bilan psychologique qui sera réalisé quelques mois plus tard révèle chez cet enfant un retard de développement associé à des particularités morphologiques ainsi que des difficultés de communication dans le cadre d'un retard global des acquisitions. 


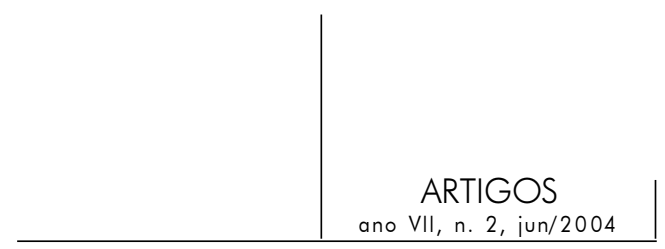

sans nom, le sujet n'est pas totalement assujetti à cet autre, agresseur, violeur, bourreau. L'expression et la reconnaissance de la honte témoignent d'une reconquête de la subjectivité, jusque-là annexée et tenue en lisière par l'enclave de honte. En effet, la honte envahit l'espace subjectif et sa répétition dans le transfert est à la mesure de "la déshérence narcissique" ressentie, pour reprendre une expression de Jacobi (2000). Autrement dit, la honte reste "honteuse" tant qu'elle n'est pas dite et mise en récit devant un tiers, en l'occurrence le clinicien dans le cadre thérapeutique. Cette honte, une fois mise en mots, sort de son enclave intrapsychique et vient s'intercaler dans la sphère intersubjective comme objet à travailler, symptôme, attribut, etc. C'est à partir du moment où elle sera introduite dans le dispositif intersubjectif qu'elle pourra être travaillée.

En conclusion, la honte et son expression scandent une problématique de subjectivation, elles indiquent et amènent une réinscription dans la temporalité. Elles permettent un changement de registre entre l'identification spéculaire et l'identification symbolique, et une capacité nouvelle à s'adresser à l'autre, le petit autre et le grand Autre.

Assoun, P.-L. Leçons psychanalytiques sur le regard et la voix. Tome 2: Figures. Paris: Anthropos, 1995.

Barazer, C. Hontes sans issue. Documents \& Débats, Bulletin Intérieur de l'Association Psychanalytique de France, n. 52, 2000.

Chemama, R. (1993). Dictionnaire de la psychanalyse. Paris: Larousse, 1995.

Dor, J. Introduction à la lecture de Lacan, 1. L'inconscient est structuré comme un langage. Paris: Denoël, 1995.

FERENCZI, S. (1932). Journal clinique. Paris: Payot, 1985.

Freud, S. (1896). Nouvelles remarques sur les psychonévroses de défense. In: Névrose, psychose et perversion. Paris: P.U.F., 1973. . (1900). L'interprétation des rêves. Paris: P.U.F., 1967. . (1905). Trois essais sur la théorie sexuelle. Paris: Gallimard, 1987. . (1914). Pour introduire le narcissisme. Paris: Gallimard, 1968. . (1915). L'inconscient. In: Métapsychologie. Paris: Gallimard, 1968. . (1921). Psychologie des foules et analyse du moi. In: Essais de psychanalyse. Paris: Payot, 1981. (1923). Le Moi et le Ça. In: Essais de psychanalyse. Paris: Payot, 1981. 


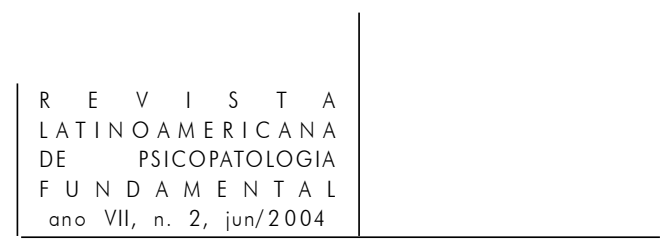

(1926). Inhibition, symptôme et angoisse. Paris: Quadrige/P.U.F., 1993.

. (1933). Nouvelles conférences d'introduction à la psychanalyse. Paris: Gallimard, 1984.

Gori, R. Le traumatisme de l'ordinaire. Colloque national de victimologie clinique"Le traumatisme sexuel et ses devenirs". Montpellier, 23 \& 24 juin 2000. Communication orale.

La Bible de Jérulasem. L'Ancien Testament. Paris: Éditions du Cerf, 1977.

JACOBI, B. Les mots et la plainte. Toulouse: Erès, 1998.

. Introduction sur un malaise supposé. Colloque du CIRPC/Université de Provence d'Aix-Marseille I et Université Louis Pasteur de Strasbourg - "Malaise dans la filiation”. Aix-en-Provence, 27 e 28 novembre 1999. Communication orale.

De la honte à la plainte. Colloque national de victimologie clinique - "Le traumatisme sexuel et ses devenirs”. Montpellier, 23 e 24 juin 2000. Communication orale.

LACAN, J. (1945a). Le stade du miroir comme formateur de la fonction du Je. In: Écrits Paris: Seuil, 1966. p. 93-100.

. (1945b). Le temps logique et l'assertion de certitude anticipée. Un nouveau sophisme. In: Écrits. Paris: Seuil, 1966. p. 197-213. . (1948). L'agressivité en psychanalyse. In: Écrits. Op. cit. p. 101-24. . (1957-1958). Le séminaire. Livre V. Les formations de l'inconscient. Paris: Seuil, 1998.

(1960). Subversion du sujet et dialectique du désir dans l'inconscient freudien. In: Écrits. Op. cit. p. 793-827.

. (1966). De nos antécédents. In: Écrits. Op. cit. p. 65-72.

. (1975-1976). Séminaire XXIII. Le sinthome. Séminaire pirate inédit.

Laplanche, J. et Pontalis, J.-B. (1967). Vocabulaire de la psychanalyse. Paris: P.U.F., 1992. LÉvy, G. Au-delà du malaise. Psychanalyse et barbaries. Toulouse: Erès, 2000.

Miollan, C. Inceste, une écoute post-traumatique. Cliniques Méditerranéennes: Exil et migrations dans la langue, Toulouse: Erès, n. 55/56, 1998.

Rycroft, C. Dictionnaire de psychanalyse. Paris: Hachette, Marabout Université, 1982. SCHNEIDER, M. Le franchissement du seuil, Freud et la thématique du regard. Cliniques Méditerranéennes: Césure du regard, Cliniques du visuel, Toulouse: Erès, n. 51/52, 1996.

Scotto di Vettimo, D. Métapsychologie et clinique de la honte: son statut, ses manifestations, son traitement psychothérapique. 2001. Thèse (Doctorat en Sciences Humaines), Université de Nice Sophia-Antipolis, Nice.

Tisseron, S. La Honte, psychanalyse d'un lien social. Paris: Dunod, 1992. 


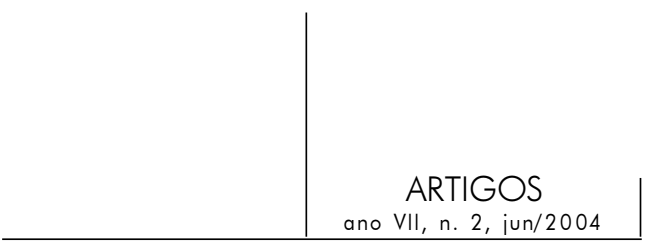

Resumos

O tratamento psicoterápico de vítimas de trauma sexual, sejam elas crianças ou adultos, geralmente revela um sentimento de vergonha. Este sentimento, esta máscara de vergonha, é, em primeiro lugar, um sentimento social, e sempre aparece como reação à maneira pela qual uma outra pessoa nos olha. No presente artigo, discutimos a hipótese de que, em tratamento psicoterápico, a expressão facial do sujeito e o reconhecimento da vergonha, longe de ser epifenômenos, constituem um suporte essencial para uma identidade que sustente e reconstrua. Isto significa que, ao invés de reduzir a vergonha a um sintoma, a mesma deve ser incluída em um entendimento mais amplo a respeito do funcionamento do psiquismo. Ser reconhecido por si mesmo e pelos outros como um sujeito "vergonhoso" é uma afirmação do eu como sujeito.

Uma situação clínica será discutida para mostrar como, em consultas postraumáticas, o reconhecimento do sentido de vergonha tem efeitos positivos sobre a dinâmica mental do sujeito.

Palavras-chave: Sentimento de vergonha, trauma sexual, narcisismo, identidade, psicoterapia

El tratamiento psicoterapéutico de niños y adultos víctimas de traumatismos sexuales hace aparecer un sentimiento de vergüenza característico. Esta prueba de vergüenza que enrojece y maquilla el rostro, es primeramente un sentimiento social: aparece siempre en reacción a la mirada del otro. La hipótesis aquí abordada es la de que en el dispositivo psicoterapéutico, la expresión y el reconocimiento de la vergüenza por parte del sujeto, lejos de ser un epifenómeno, constituye un punto de apoyo esencial en la afirmación y la reconstrucción de la identidad. Se trata de no reducir la vergüenza a un síntoma sino de incluirla en una comprensión más global del funcionamiento psíquico: reconocerse y hacerse reconocer sujeto avergonzado es ya afirmarse sujeto.

La evocación de una situación clínica será propuesta para describir cómo llevar en cuenta la prueba de la vergüenza en una escucha postraumática tiene efectos positivos sobre la dinámica psíquica del sujeto.

Palabras clave: Sentimiento de vergüenza, trauma sexual, narcisismo, identidad, psicoterapia

The psychotherapeutic treatment of child and adult victims of sexual trauma reveals a characteristic feeling of shame. This sense of shame, this mask or blushing face, is first and foremost a social feeling: it always appears as a reaction to how someone else looks at us. Here we discuss the hypothesis that in psychotherapeutic treatment, the subject's expression and recognition of shame, far from being 


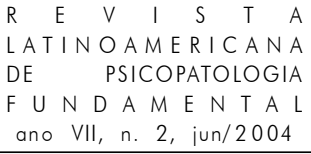

epiphenomenona, constitute an essential aid to affirming and reconstructing identity. This means that rather than reducing shame to a symptom, it should be included in a more global understanding of how the psyche functions. Recognising oneself and being recognised as a shameful subject is an affirmation of the self as subject.

A clinical situation will be discussed to show how, in post-traumatic consultation, taking account of the sense of shame has positive effects on the subject's mental dynamics.

Key words: Feeling of shame, sexual trauma, narcissism, identity, psychotherapy 